\title{
L $^{2}$-TORSION, THE MEASURE-THEORETIC DETERMINANT CONJECTURE, AND UNIFORM MEASURE EQUIVALENCE
}

\author{
WOLFGANG LÜCK, ROMAN SAUER, AND CHRISTIAN WEGNER
}

\begin{abstract}
We show an invariance result for the $L^{2}$-torsion of groups under uniform measure equivalence provided a measure-theoretic version of the determinant conjecture holds. The measure-theoretic determinant conjecture is discussed and, for instance, proved for Bernoulli actions of residually amenable groups.
\end{abstract}

\section{INTRODUCTION}

Gaboriau [13] introduced $L^{2}$-Betti numbers of measured equivalence relations and proved that two measure equivalent countable groups have proportional $L^{2}$-Betti numbers. This notion turned out to have many important applications in recent years, most notably through the work of Popa [21].

In the present paper we study another well known $L^{2}$-invariant of a discrete group $G$, the $L^{2}$-torsion $\rho^{(2)}(G)$, with regard to measure equivalence. The $L^{2}$-torsion (Definition 2.17) is only defined if all the $L^{2}$-Betti numbers of $G$ vanish and the determinant conjecture (see Definition 2.16) - an integral relative of the Connes' embedding problem (see Remark 3.3)holds for $G$. The determinant conjecture is intensively studied $[1,4,25]$, and there is no counterexample known. Notably, all sofic groups satisfy the determinant conjecture [7].

The notion of measure equivalence was introduced by Gromov [15, 0.5.E] and, for the first time, gained prominence in the work of Furman [10, Definition 1.1]:

Definition 1.1. Two countable groups $G$ and $H$ are called measure equivalent with index $c=I(G, H)>0$ if there exists a non-trivial standard measure space $(\Omega, \mu)$ on which $G \times H$ acts such that the restricted actions of $G=G \times\{1\}$ and $H=\{1\} \times H$ have measurable fundamental domains $X \subset \Omega$ and $Y \subset \Omega$, with $\mu(X)<\infty, \mu(Y)<\infty$, and $c=\mu(X) / \mu(Y)$. The space $(\Omega, \mu)$ is called a measure coupling between $G$ and $H$ (of index $c$ ).

Evidence for the following conjectural analog (compare [20, Question 7.35 on p. 313]) of the aforementioned result by Gaboriau comes from computations and the formal similarities between $\rho^{(2)}(G)$ and the Euler characteristic of $G$.

Conjecture 1.2. Let $G$ and $H$ be countable groups such that all the $L^{2}$-Betti numbers of $G$ and $H$ vanish. Assume that both $G$ and $H$ admit finite $C W$-models for their classifying spaces. Then $G$ and $H$ satisfy the determinant conjecture. If $G$ and $H$ are measure equivalent with index $c=I(G, H)>0$, then

$$
\rho^{(2)}(G)=c \cdot \rho^{(2)}(H) .
$$

2000 Mathematics Subject Classification. Primary: 57Q10; Secondary: 20F65, 37A20, 46L85.

Key words and phrases. $L^{2}$-torsion, measure equivalence, orbit equivalence.

We gratefully acknowledge financial support by the Sonderforschungsbereich 478 Geometrische Strukturen in der Mathematik and by the Max-Planck-Forschungspreis and the Leibniz-Preis of the first author. 
If one attempts to solve the conjecture by eventually reducing it to homotopy invariance, which is done in [13] for $L^{2}$-Betti numbers (see [23] for another approach), then one encounters at least two difficulties that do not appear for $L^{2}$-Betti numbers: one is the need of a measure-theoretic version of the determinant conjecture (Definition 1.6 below), the other is that the Fuglede-Kadison determinant lacks a continuity property that is obvious for the trace (discussed in Section 5). The latter difficulty disappears if we restrict to uniformly measure equivalent groups in Conjecture 1.2.

The proof of the invariance of the $L^{2}$-torsion under uniform measure equivalence, which is the main topic of this paper, is nevertheless much more involved than the one of the invariance of $L^{2}$-Betti numbers under uniform measure equivalence.

Definition 1.3. Two countable groups are uniformly measure equivalent of index $c=$ $I(G, H)$ if there exists a measure coupling $(\Omega, \mu)$ between $G$ and $H$ of index $c$ with a measurable $G$-fundamental domain $X$ and measurable $H$-fundamental domain $Y$ such that the following two conditions hold:

(1) for every $g \in G$ there is a finite subset $H(g) \subset H$ such that $g Y \subset H(g) Y$ up to $\mu$-null sets, and

(2) for every $h \in H$ there is a finite subset $G(h) \subset G$ such that $h X \subset G(h) X$ up to $\mu$-null sets.

Uniform measure equivalence was introduced by Shalom and studied in the context of quasi-isometry of amenable groups by Shalom and the second author [24, 26]. Uniform measure equivalence is much more restrictive and geometric than measure equivalence. Here are important examples:

Example 1.4.

(1) Two uniform lattices in the same locally compact, second countable Hausdorff group are uniformly measure equivalent.

(2) Two finitely generated amenable groups are uniformly measure equivalent if and only if they are quasi-isometric [22, Lemma 2.25; 26, Theorem 2.1.7].

Next we introduce a measure-theoretic version of the determinant conjecture. Some definitions are in order:

Let $\mathcal{R}$ be a standard equivalence relation on a standard probability space $(X, \mu)$ with an invariant measure $\mu$ in the sense of $[8$, Section 2$]$. We call an equivalence relation with these properties just a measured equivalence relation. We call an action of a countable group $G$ on a standard probability space $(X, \mu)$ standard if it is measurable and $\mu$ is $G$-invariant. Every measured equivalence relation on $(X, \mu)$ is the orbit equivalence relation of some standard action of a countable group on $(X, \mu)[8$, Theorem 1$]$.

Definition 1.5. The groupoid ring of $\mathcal{R}$ is, as an additive group, defined as

$$
\begin{aligned}
\mathbb{C} \mathcal{R}=\left\{f: \mathcal{R} \rightarrow \mathbb{C} \mid \exists N \in \mathbb{N}: f^{-1}\left(\mathbb{C}^{\times}\right) \cap\{x\}\right. & \times X \text { and } f^{-1}\left(\mathbb{C}^{\times}\right) \cap X \times\{x\} \text { have } \\
\text { cardinality at most } N \text { a.e. }\} & \subset L^{\infty}(\mathcal{R}, \mathbb{C}) .
\end{aligned}
$$

endowed with the following multiplication, involution, and trace, respectively:

(1) $(f g)(x, y)=\sum_{z \sim x} f(x, z) g(z, y)$,

(2) $f^{*}(x, y)=f(y, x)$,

(3) $\operatorname{tr}_{\mathcal{N}(\mathcal{R})}(f)=\int_{X} f(x, x) d \mu(x)$. 
The integral groupoid ring is $\mathbb{Z} \mathcal{R}=\mathbb{C} \mathcal{R} \cap L^{\infty}(\mathcal{R}, \mathbb{Z})$. The finite von Neumann algebra of $\mathcal{R}\left[9\right.$, Section 2], which contains $\mathbb{C} \mathcal{R}$ as a weakly dense subset and to which $\operatorname{tr}_{\mathcal{N}(\mathcal{R})}$ extends as a finite trace, is denoted by $\mathcal{N}(\mathcal{R})$. If $\mathcal{R}$ is the orbit equivalence relation of a standard action $G \curvearrowright X$, we will also use the notation $\mathcal{N}(G \curvearrowright X)$ instead of $\mathcal{N}(\mathcal{R})$.

Definition 1.6. Let $\mathcal{R}$ be a standard equivalence relation and $G$ be a countable group. We say that $\mathcal{R}$ satisfies the measure-theoretic determinant conjecture (abbreviated as $M D C$ ) if the (generalized) Fuglede-Kadison determinant of every matrix $A \in M(m \times n, \mathbb{Z} \mathcal{R})$ satisfies

$$
\operatorname{det}_{\mathcal{N}(\mathcal{R})}(A) \geq 1
$$

A standard action of $G$ satisfies MDC if its orbit equivalence relation satisfies MDC. The group $G$ satisfies MDC if every essentially free, standard action of $G$ satisfies MDC.

Conjecture 1.7 (Measure Theoretic Determinant Conjecture). Every standard equivalence relation satisfies the measure-theoretic determinant conjecture.

The following theorem, actually a stronger version thereof, is proved in Section 3.

Theorem 1.8. Let $G$ be a countable group.

(1) Countable amenable groups satisfy $M D C$.

(2) If $G$ satisfies $M D C$, then every subgroup of $G$ satisfies $M D C$.

(3) If $G$ satisfies $M D C$, then every amenable extension of $G$ satisfies $M D C$.

(4) Let $1 \rightarrow K \rightarrow G \rightarrow Q \rightarrow 1$ be a group extension such that $K$ is finite. If $G$ satisfies $M D C$, then $Q$ satisfies $M D C$.

(5) Assume that $G=\operatorname{colim}_{i \in I} G_{i}$ where $\left(G_{i}\right)_{i \in I}$ is a directed system of countable groups (whose structure maps are not necessarily injective). If every $G_{i}$ satisfies $M D C$, then $G$ satisfies $M D C$.

(6) Bernoulli actions of countable residually amenable groups satisfy $M D C$.

Theorem 1.9. Let $G$ and $H$ be measure equivalent groups. If $G$ satisfies $M D C$, then $H$ satisfies the determinant conjecture.

The preceding theorem is proved in Section 4. In the same section we also prove the following theorem as a first step towards Conjecture 1.2. The conclusion about the vanishing of the $L^{2}$-Betti numbers of $H$ below is of course due to the corresponding theorem of Gaboriau [13], which we built in for a clean formulation.

Theorem 1.10. Let $G$ and $H$ be groups that admit finite $C W$-models for their classifying spaces. Assume that all the $L^{2}$-Betti numbers of $G$ vanish and $G$ satisfies $M D C$. If $G$ and $H$ are uniformly measure equivalent with index $c=I(G, H)>0$, then all the $L^{2}$-Betti numbers of $H$ vanish, $H$ satisfies the determinant conjecture, and

$$
\rho^{(2)}(G)=c \cdot \rho^{(2)}(H) .
$$

Example 1.4 yields the following corollary:

Corollary 1.11. Let $G$ and $H$ be amenable groups that admit finite $C W$-models for their classifying spaces. If $G$ and $H$ are quasi-isometric, then

$$
\rho^{(2)}(G)=0 \Longleftrightarrow \rho^{(2)}(H)=0 .
$$

We emphasize that there is the conjecture that the $L^{2}$-torsion vanishes for all infinite amenable groups. In [28] it is shown that $\rho^{(2)}(G)=0$ if $G$ contains an infinite elementary amenable normal subgroup and has a finite model of its classifying space. 


\section{BACKGROUND IN $L^{2}$-INVARIANTS AND ORBIT EQUIVALENCE RELATIONS}

2.1. Uniform orbit equivalence. The notion of orbit equivalence has its roots in the pioneering work of Dye $[5,6]$. We recall it here:

Definition 2.1. Two standard actions $G \curvearrowright\left(X, \mu_{X}\right)$ and $H \curvearrowright\left(Y, \mu_{Y}\right)$ are called weakly orbit equivalent with index $c=I(G, H)>0$ if there are measurable subsets $A \subset X, B \subset Y$ and a measurable isomorphism $f: A \rightarrow B$ such that

(1) $G \cdot A=X$ and $H \cdot B=Y$ up to null sets,

(2) $\frac{1}{\mu_{X}(A)} f_{*}\left(\left.\mu_{X}\right|_{A}\right)=\left.\frac{1}{\mu_{Y}(B)} \mu_{Y}\right|_{B}$

(3) $f(G \cdot x \cap A)=H \cdot f(x) \cap B$ for a.e. $x \in A$, and

(4) $c=\mu_{X}(A) / \mu_{Y}(B)$.

If $\mu_{X}(A)=\mu_{Y}(B)=1$, then we call the actions orbit equivalent. The map $f$ is called a weak orbit equivalence or orbit equivalence, respectively.

The following modification of orbit equivalence was introduced in [22] (under the name bounded orbit equivalence).

Definition 2.2. Two standard actions $G \curvearrowright\left(X, \mu_{X}\right)$ and $H \curvearrowright\left(Y, \mu_{Y}\right)$ are called uniformly weakly orbit equivalent with index $c=I(G, H)$ if there exists a weak orbit equivalence $f: A \rightarrow B$ of index $c$ as in Definition 2.1 that satisfies the following additional properties:

(1) There exist finite subsets $F_{A} \subset G$ and $F_{B} \subset H$ such that $F_{A} \cdot A=X$ and $F_{B} \cdot B=Y$ up to null sets,

(2) For every $g \in G$ there is a finite subset $F(g) \subset H$ such that $f(g x) \in F(g) \cdot f(x)$ for a.e. $x \in A \cap g^{-1} \cdot A$.

(3) For every $h \in H$ there is a finite subset $F(h) \subset G$ such that $f^{-1}(h y) \in F(h) \cdot f^{-1}(y)$ for a.e. $y \in B \cap h^{-1} \cdot B$.

The following theorem is proved in [11, Theorem 3.3] (see also [22, Theorem 2.33] for the uniform version).

Theorem 2.3. Two countable groups $G$ and $H$ are (uniformly) measure equivalent with respect to a measure coupling of index $c>0$ if and only if there exist essentially free, standard actions $G$ and $H$ that are (uniformly) weakly orbit equivalent of index $c$.

\subsection{Algebras associated to groups and equivalence relations.}

Definition 2.4. Let $G \curvearrowright(X, \mu)$ be a standard action. The crossed product ring $L^{\infty}(X, \mathbb{C}) *$ $G$ is the free $L^{\infty}(X, \mathbb{C})$-module with $G$ as $L^{\infty}(X, \mathbb{C})$-basis. Here $L^{\infty}(X, \mathbb{C})$ denotes the ring of equivalence classes of essentially bounded measurable functions $X \rightarrow \mathbb{C}$. The multiplication is given by

$$
\left(\sum_{g \in G} r_{g} \cdot g\right) \cdot\left(\sum_{g \in G} s_{g} \cdot g\right)=\sum_{g \in G}\left(\sum_{\substack{g_{1}, g_{2} \in G \\ g_{1} g_{2}=g}} r_{g_{1}} \cdot\left(s_{g_{2}} \circ m_{g_{1}-1}\right)\right) \cdot g
$$

with $m_{g}: X \rightarrow X, x \mapsto g x$. The crossed product ring $L^{\infty}(X, \mathbb{Z}) * G$ is defined analogously using the ring of equivalence classes of essentially bounded measurable functions $X \rightarrow \mathbb{Z}$.

Remark 2.5 (compare [22, Sections 1.2 and 1.3]). Let $G \curvearrowright(X, \mu)$ be an essentially free, standard action and $\mathcal{R}$ be its orbit equivalence relation. The rings $L^{\infty}(X, \mathbb{Z}) * G$ and 
$L^{\infty}(X, \mathbb{C}) * G$ embed as subrings into $\mathbb{Z} \mathcal{R}$ or $\mathbb{C} \mathcal{R}$, respectively. Let $i_{g}: X \rightarrow \mathcal{R}$ be the map $i_{g}(x)=\left(x, g^{-1} x\right)$. We obtain a (multiplicative) isomorphism

$L^{\infty}(X, \mathbb{C}) * G \cong\left\{f \in \mathbb{C} \mathcal{R} \mid f \circ i_{g}=0 \in L^{\infty}(X, \mathbb{C})\right.$ for all but finitely many $\left.g \in G\right\} \subset \mathbb{C} \mathcal{R}$ given by $\sum_{g \in G} r_{g} \cdot g \mapsto f$ with $f\left(x, g^{-1} x\right)=r_{g}(x)$. There is an analogous statement for integral (instead of complex) coefficients. In particular, we obtain a trace-preserving inclusion $\mathbb{C} G \hookrightarrow \mathbb{C} \mathcal{R}$; this inclusion extends to the von Neumann algebras $\mathcal{N}(G)$ of $G$ and $\mathcal{N}(\mathcal{R})$ of $\mathcal{R}$.

The following lemmas about the crossed product of a standard action $G \curvearrowright X$ are rather easy to verify.

Lemma 2.6. $L^{\infty}(X, \mathbb{Z}) * G$ is flat over $\mathbb{Z} G$.

Proof. This follows from the fact that $L^{\infty}(X, \mathbb{Z})$ is torsionfree and the isomorphism

$$
L^{\infty}(X, \mathbb{Z}) * G \otimes_{\mathbb{Z} G} M \cong L^{\infty}(X, \mathbb{Z}) \otimes_{\mathbb{Z}} M .
$$

Recall that that an idempotent $p$ in a ring $R$ is called full if the additive group generated by elements of the form $r p r^{\prime}, r, r^{\prime} \in R$, is $R$. If $p \in R$ is full, then the rings $p R p$ and $R$ are Morita equivalent. This implies e.g. that $P$ is a finitely generated projective $R$-module if and only if $p P$ is a finitely generated projective $p R p$-module [17, (18.30B) on p. 490]. Note also that if $R \subset S$ is a unital subring and $p \in R$ is a full idempotent in $R$, then $p$ is also a full idempotent in $S$.

Remark 2.7. Let $\mathcal{R}$ be the orbit equivalence relation of a standard action of $G$ on $(X, \mu)$. Let $A \subset X$ be a subset. We denote the restricted equivalence relation by $\left.\mathcal{R}\right|_{A}=\mathcal{R} \cap A \times A$. One has

$$
\left.\mathbb{Z} \mathcal{R}\right|_{A}=\chi_{A} \mathbb{Z} \mathcal{R} \chi_{A},
$$

where $\chi_{A}$ is the characteristic function of $A$. Similarly, $\mathcal{N}\left(\left.\mathcal{R}\right|_{A}\right)=\chi_{A} \mathcal{N}(\mathcal{R}) \chi_{A}$.

Lemma 2.8 ([22, Lemma 4.21]). Let $A \subset X$ be a measurable subset such that finitely many $G$-translates of $A$ cover $X$ up to null sets. Then the characteristic function $\chi_{A}$ is a full idempotent in $\chi_{A} L^{\infty}(X, \mathbb{Z}) * G \chi_{A}$.

Lemma 2.9 ([22, Lemma 4.23]). Let $f: A \rightarrow B$ be an orbit equivalence between essentially free, standard actions of $G$ on $\left(X, \mu_{X}\right)$ and $H$ on $\left(Y, \mu_{Y}\right)$. Let $\mathcal{R}_{1}$ and $\mathcal{R}_{2}$ be the orbit equivalence relations of $G \curvearrowright X$ and $H \curvearrowright Y$, respectively.

(1) The isomorphism $\left.\left.\mathcal{R}_{1}\right|_{A} \stackrel{f \times f}{\longrightarrow} \mathcal{R}_{2}\right|_{B}$ induces the trace-preserving $*$-isomorphism

$$
f^{*}:\left.\left.\mathbb{Z} \mathcal{R}_{2}\right|_{B} \rightarrow \mathbb{Z} \mathcal{R}_{1}\right|_{A}, \phi \mapsto \phi \circ(f \times f) .
$$

Thus it extends to an isomorphism $\mathcal{N}\left(\left.\mathcal{R}_{2}\right|_{B}\right) \rightarrow \mathcal{N}\left(\left.\mathcal{R}_{1}\right|_{A}\right)$.

(2) If $f$ is uniform, the isomorphism $f^{*}$ restricts to an isomorphism

$$
\chi_{B} L^{\infty}(Y, \mathbb{Z}) * H \chi_{B} \rightarrow \chi_{A} L^{\infty}(X, \mathbb{Z}) * G \chi_{A}
$$

of the embedded subrings.

Convention 2.10. Let $G \curvearrowright X$ be a standard action, $A \subset X$ be a measurable subset, and $\mathcal{R}$ be the orbit equivalence relation of the action. We introduce some equivalent notations: Instead of $\mathcal{N}(\mathcal{R})$ and $\mathcal{N}(\mathcal{R} \mid A)$ we also write $\mathcal{N}(G \curvearrowright X)$ and $\mathcal{N}\left(\left.G \curvearrowright X\right|_{A}\right)$, respectively. For the $G N S$-construction $l^{2}\left(\mathcal{N}\left(\left.\mathcal{R}\right|_{A}\right)\right)$ of $\mathcal{N}\left(\left.\mathcal{R}\right|_{A}\right)$ we write $L^{2}\left(\left.\mathcal{R}\right|_{A}\right)$ or $L^{2}\left(\left.G \curvearrowright X\right|_{A}\right)$. 
2.3. The Fuglede-Kadison determinant and $L^{2}$-torsion. In the sequel, let $\mathcal{A}$ be a finite von Neumann algebra with $\operatorname{trace}_{\operatorname{tr}} \operatorname{tr}_{\mathcal{A}} \rightarrow \mathbb{C}$. Our main examples are the group von Neumann algebra $\mathcal{N}(G)$ of a group $G$ and the von Neumann algebra $\mathcal{N}(\mathcal{R})$ of a standard equivalence relation. A Hilbert space with a (left) $\mathcal{A}$-action that embeds isometrically and equivariantly as a closed subspace into a Hilbert sum of copies of $l^{2}(\mathcal{A})$ is a called a Hilbert $\mathcal{A}$-module. A bounded $\mathcal{A}$-equivariant operator between Hilbert $\mathcal{A}$-modules is called a morphism of Hilbert $\mathcal{A}$-modules. The $\operatorname{trace} \operatorname{tr}_{\mathcal{A}}$ extends in a natural way to positive morphisms of Hilbert $\mathcal{A}$-modules. Further, every Hilbert $\mathcal{A}$-module $H$ has a real-valued dimension $\operatorname{dim}_{\mathcal{A}}(H) \in[0, \infty]$. This dimension satisfies, for example, $\operatorname{dim}_{\mathcal{A}}\left(l^{2}(\mathcal{A}) p\right)=\operatorname{tr}_{\mathcal{A}}(p)$ for any projection $p \in \mathcal{A}$. We refer to $[20$, Chapter 6$]$ for more information. The following convention is adopted:

Convention 2.11. The $n \times n$-matrices $M(n \times n, \mathcal{A})$ over $\mathcal{A}$ are again a von Neumann algebra. We equip $M(n \times n, \mathcal{A})$ with the unnormalized trace $\operatorname{tr}_{M(n \times n, \mathcal{A})}(A)=\sum_{i=1}^{n} \operatorname{tr}_{\mathcal{A}}\left(A_{i i}\right)$. If the context is clear, we just write $\operatorname{tr}_{\mathcal{A}}$ instead of $\operatorname{tr}_{M(n \times n, \mathcal{A})}$. There is a one-to-one correspondence between $A \in M(n \times n, \mathcal{A})$ and (left)-A-equivariant bounded operators $l^{2}(\mathcal{A})^{n} \rightarrow l^{2}(\mathcal{A})^{n}$ via right matrix multiplication. If we want to stress the point of view of $A$ as a bounded operator, we also use the notation $r_{A}$ for the right multiplication on $l^{2}(\mathcal{A})^{n}$.

We remind the reader of the definition of the spectral density function and the FugledeKadison determinant: Let $f: U \rightarrow V$ be a morphism of Hilbert $\mathcal{A}$-modules of finite dimension. Denote by $\left\{E_{\lambda}^{f^{*} f}: U \rightarrow U \mid \lambda \in \mathbb{R}\right\}$ the family of spectral projections of the positive endomorphism $f^{*} f$. The spectral projections have the properties $\|f(u)\| \leq \lambda \cdot\|u\|$ for $u \in \operatorname{im}\left(E_{\lambda^{2}}^{f^{*} f}\right)$ and $\|f(u)\|>\lambda \cdot\|u\|$ for $0 \neq u \in \operatorname{ker}\left(E_{\lambda^{2}}^{f^{*} f}\right)$. The spectral density function of $f$ is defined as

$$
F(f): \mathbb{R} \rightarrow[0, \infty), \lambda \mapsto \operatorname{tr}_{\mathcal{A}}\left(E_{\lambda^{2}}^{f^{*} f}\right) .
$$

The spectral density function $F(f)$ is monotonous and right-continuous.

Definition 2.12 ([20, Definition 3.11 on p. 127]). Let $f: U \rightarrow V$ be a morphism of Hilbert $\mathcal{A}$-modules of finite dimensions. The Fuglede-Kadison determinant or just determinant of $f$ is defined as

$$
\operatorname{det}_{\mathcal{A}}(f)= \begin{cases}\exp \left(\int_{0^{+}}^{\infty} \ln (\lambda) d F(f)(\lambda)\right) & \text { if } \int_{0^{+}}^{\infty} \ln (\lambda) d F(f)(\lambda)>-\infty \\ 0 & \text { otherwise. }\end{cases}
$$

Here the integral is understood to be the Lebesgue-Stieltjes integral with respect to $F(f)$.

The main properties of this determinant are described in [20, Theorem 3.14].

Remark 2.13 (Induction). Let $\mathcal{A} \hookrightarrow \mathcal{B}$ be a trace-preserving inclusion of finite von Neumann algebras. Let $f: l^{2}(\mathcal{A})^{m} \rightarrow l^{2}(\mathcal{A})^{n}$ be a morphism of Hilbert $\mathcal{A}$-modules. Then $f$ is given by right multiplication $r_{A}$ with an $m \times n$-matrix over $\mathcal{A}$. The morphism of Hilbert $\mathcal{B}$-modules $l^{2}(\mathcal{B})^{m} \rightarrow l^{2}(\mathcal{B})^{n}$ defined by right multiplication with the same matrix is denoted by $\operatorname{ind}_{\mathcal{A}}^{\mathcal{B}}(f)$. It is obvious that

$$
\operatorname{tr}_{\mathcal{B}}\left(P\left(\operatorname{ind}_{\mathcal{A}}^{\mathcal{B}}(f)\right)\right)=\operatorname{tr}_{\mathcal{A}}(P(f))
$$

for any complex polynomial $P$ and $m=n$. Normality of the trace yields that $F\left(\operatorname{ind}_{\mathcal{A}}^{\mathcal{B}}(f)\right)=$ $F(f)$, hence

$$
\operatorname{det}_{\mathcal{B}}\left(\operatorname{ind}_{\mathcal{A}}^{\mathcal{B}}(f)\right)=\operatorname{det}_{\mathcal{A}}(f)
$$


Remark 2.14 (Restriction). Let $p \in \mathcal{A}$ be a projection. Then $p \mathcal{A} p$ is a finite von Neumann algebra with normalized $\operatorname{trace} \operatorname{tr}_{p \mathcal{A} p}=\frac{1}{\operatorname{tr}_{\mathcal{A}}(p)} \operatorname{tr}_{\mathcal{A}}$ [3, Prop. 1 on p. 17]. Let $f: U \rightarrow V$ be a morphism of finitely generated Hilbert $\mathcal{A}$-modules. The morphism $f^{*} f$ decomposes as an orthogonal sum

$$
p U \oplus(1-p) U \stackrel{\left.\left.f^{*} f\right|_{p U} \oplus f^{*} f\right|_{(1-p) U}}{\longrightarrow} p U \oplus(1-p) U .
$$

Since spectral calculus respects orthogonal sums, we obtain that $\operatorname{im}\left(E_{\lambda^{2}}^{\left.f^{*} f\right|_{p U}}\right)=p \operatorname{im}\left(E_{\lambda^{2}}^{f^{*} f}\right)$. Viewing $\left.f\right|_{p U}$ as a morphism of Hilbert $p \mathcal{A} p$-modules, we obtain that $F(f)=\operatorname{tr}_{\mathcal{A}}(p) F\left(\left.f\right|_{p U}\right)$ provided $p$ is full [16, Proposition 2.2.6 vii) on p. 26]. From this we conclude that, if $\operatorname{det}_{\mathcal{A}}(f)>0$, then

$$
\ln \operatorname{det}_{\mathcal{A}}(f)=\operatorname{tr}_{\mathcal{A}}(p) \cdot \ln \operatorname{det}_{p \mathcal{A} p}\left(\left.f\right|_{p U}\right) .
$$

Definition 2.15. Let $C_{*}$ a Hilbert $\mathcal{A}$-chain complex. Suppose that

(1) $C_{*}$ is dim-finite, i.e. $\operatorname{dim}_{\mathcal{A}}\left(C_{n}\right)<\infty$ for all $n \in \mathbb{Z}$ and there exists $N \in \mathbb{N}$ such that $C_{n}=0$ if $n<0$ or $n>N$,

(2) $b_{n}^{(2)}\left(C_{*}\right)=0$ for all $n \in \mathbb{Z}$,

(3) $\operatorname{det}_{\mathcal{A}}\left(c_{n}\right)>0$ for all $n \in \mathbb{Z}$.

We define its $L^{2}$-torsion by

$$
\rho^{(2)}\left(C_{*}\right)=-\sum_{n \in \mathbb{Z}}(-1)^{n} \ln \left(\operatorname{det}_{\mathcal{A}}\left(c_{n}\right)\right) \in \mathbb{R} .
$$

The following conjecture is true for all sofic groups [7]; no example of a group that is not sofic is known.

Definition 2.16 ([20, Conjecture 13.2 on p. 454]). We say that the group $G$ satisfies the determinant conjecture or is of determinant class if the Fuglede-Kadison determinant of every matrix $A \in M(m \times n, \mathbb{Z} G)$ satisfies

$$
\operatorname{det}_{\mathcal{N}(G)}(A) \geq 1
$$

Let $X$ be a finite CW-complex with vanishing $L^{2}$-Betti numbers such that $G=\pi_{1}(X)$ satisfies the determinant conjecture. We define the $L^{2}$-torsion of $X$ as

$$
\rho^{(2)}(\widetilde{X})=\rho^{(2)}\left(l^{2}(G) \otimes_{\mathbb{Z} G} C_{*}^{\text {cell }}(\widetilde{X})\right) .
$$

Since the differentials in the cellular chain complex $C_{*}^{\text {cell }}(\widetilde{X})$ are matrices over $\mathbb{Z} G$ with respect to cellular bases and thus have positive determinant, this definition is justified. If $G$ is of determinant class, then this definition only depends on the homotopy type of $X[20$, Lemma 13.6 on p. 456].

Definition 2.17. Let $G$ be a group that admits a finite CW-model $X$ of its classifying space $B G$. Suppose that the group $G$ satisfies the determinant conjecture and that all its $L^{2}$-Betti numbers vanish. Then we define the $L^{2}$-torsion of $G$ as $\rho^{(2)}(G)=\rho^{(2)}(\widetilde{X})$.

\section{The measure-theoretic DETERminant CONJECture}

The goal of this section is to prove Theorem 1.8; we actually prove the following slightly stronger formulation:

Theorem 3.1. Let $G$ be a countable group and $H \subset G$ a subgroup.

(1) The trivial group satisfies $M D C$. 
(2) If $G$ satisfies $M D C$, then $H$ satisfies $M D C$.

(3) Let $H \subset G$ be a normal subgroup such that the quotient $G / H$ is amenable. Let $G \curvearrowright X$ be a standard action. If $H \curvearrowright X$ satisfies $M D C$, then $G \curvearrowright X$ satisfies $M D C$.

(4) Let $1 \rightarrow K \rightarrow G \rightarrow Q \rightarrow 1$ be a group extension such that $K$ is finite. If $G$ satisfies $M D C$, then $Q$ satisfies $M D C$.

(5) Assume that $G=\operatorname{colim}_{i \in I} G_{i}$ where $\left(G_{i}\right)_{i \in I}$ is a directed system (with not necessarily injective structure maps) of countable groups. If every $G_{i}$ satisfies $M D C$, then $G$ satisfies $M D C$.

(6) Let $(X, \mu)$ be a standard probability space. Suppose that $G$ is a directed limit of countable groups $G=\lim _{i \in I} G_{i}$ such that the shift action of $G_{i}$ on the product space $(X, \mu)^{G_{i}}$ satisfies $M D C$. Then the shift action of $G$ on $(X, \mu)^{G}$ satisfies $M D C$.

Actions as in (6) are called Bernoulli actions. The reason that we restrict to Bernoulli actions in (6) is that we do not know how to approximate an arbitrary $G$-action by actions of the groups $G_{i}$. The remainder of this section is devoted to the proof of the theorem above.

3.1. The approximation lemma. The proof of the next lemma is essentially the same as in the special case of group von Neumann algebras. Such proofs are given in [18, Lemma 2.5; 20, Theorem 13.19 on p. 461; 25, Section 6].

Lemma 3.2. Let $\mathcal{A}, \mathcal{A}_{i}(i \in I)$ be finite von Neumann algebras with $I$ a directed set. Let $A \in M\left(d \times d^{\prime} ; \mathcal{A}\right)$ and $A_{i} \in M\left(d_{i} \times d_{i}^{\prime} ; \mathcal{A}_{i}\right)$ be matrices with the following properties where $\Delta, \Delta_{i}$ are defined as $\Delta:=A A^{*} \in M(d \times d ; \mathcal{A}), \Delta_{i}:=A_{i} A_{i}^{*} \in M\left(d_{i} \times d_{i} ; \mathcal{A}_{i}\right)$ :

(1) $\operatorname{det}_{\mathcal{A}_{i}}\left(A_{i}\right) \geq 1$,

(2) there exists a constant $K>0$ with $\left\|r_{\Delta}\right\| \leq K$ and $\left\|r_{\Delta_{i}}\right\| \leq K$,

(3) $\lim _{i \in I} \frac{\operatorname{tr}_{\mathcal{A}_{i}}\left(\Delta_{i}^{m}\right)}{d_{i}}=\frac{\operatorname{tr}_{\mathcal{A}}\left(\Delta^{m}\right)}{d}$ for all $m \in \mathbb{N}$.

Then $\lim _{i \in I} \operatorname{dim}_{\mathcal{A}_{i}}\left(\operatorname{ker} A_{i}\right)=\operatorname{dim}_{\mathcal{A}}(\operatorname{ker} A)$ and $\operatorname{det}_{\mathcal{A}}(A) \geq 1$.

Remark 3.3. In Connes' pioneering paper [2] the question was raised whether every finite von Neumann algebra embeds into an ultraproduct of the hyperfinite $\mathrm{II}_{1}$-factor (nowadays referred to as the Connes embedding problem). If the Connes embedding problem has a positive answer for the finite von Neumann algebra $\mathcal{A}$, then for every self-adjoint $\Delta \in \mathcal{A}$ there is a sequence of matrices $\Delta_{i} \in M\left(d_{i} \times d_{i}, \mathbb{C}\right)$ that satisfies (3) of Lemma 3.2. If the $\Delta_{i}$ have only integral entries, then $\operatorname{det}_{\mathcal{A}}(\Delta) \geq 1$. In that regard the determinant conjecture is an integral relative of the Connes embedding problem.

The following lemma is often helpful for verifying the second condition in Lemma 3.2. We omit its proof which is essentially the same as in [20, Proof of Lemma 13.33 on p. 466].

Lemma 3.4. Let $G \curvearrowright X$ be a standard action and $A \in M\left(d \times d^{\prime} ; L^{\infty}(X, \mathbb{C}) * G\right)$. For an element $f=\sum_{g \in G} f_{g} \cdot g \in L^{\infty}(X, \mathbb{C}) * G$, let $\|f\|_{\infty}=\sum_{g \in G}\left\|f_{g}\right\|_{\infty}$. Then:

$$
\left\|r_{A}\right\| \leq d \cdot d^{\prime} \cdot \max _{k, l}\left\|A_{k, l}\right\|_{\infty} .
$$

\subsection{Some reductions used in the proof.}

Lemma 3.5. Let $G \curvearrowright(X, \mu)$ be an essentially free, standard action of a countable group $G$. Let $\mathcal{R}$ be its orbit equivalence relation on $X$. Assume that $\operatorname{det}_{\mathcal{N}(\mathcal{R})}(A) \geq 1$ for every matrix $A \in M\left(d \times d^{\prime} ; L^{\infty}(X, \mathbb{Z}) * G\right)$. Then $\mathcal{R}$ satisfies $M D C$. 
Proof. Let $A \in M\left(d \times d^{\prime} ; \mathbb{Z} \mathcal{R}\right)$. Choose an enumeration $G=\left\{g_{1}, g_{2}, \ldots\right\}$. We define an increasing sequence $\left(X_{n}\right)_{n \geq 1}$ of measurable subsets of $X$ by

$$
X_{n}=\left\{x \in X \mid A_{i j}\left(x, g_{m} x\right)=0 \text { for } m>n \text { and all } 1 \leq i \leq d, 1 \leq j \leq d^{\prime}\right\} .
$$

Obviously, $\mu\left(X_{n}\right) \rightarrow 1$. Set $A_{n}=\chi_{X_{n}} A$. Then $A_{n} \in M\left(d \times d^{\prime} ; L^{\infty}(X, \mathbb{Z}) * G\right)$ and $\left\|r_{A_{n} A_{n}^{*}}\right\|=$ $\left\|r_{\chi_{X_{n}} A A^{*} \chi_{X_{n}}}\right\| \leq\left\|r_{A A^{*}}\right\|$. By the continuity of the trace and the trace property, we obtain that

$$
\operatorname{tr}_{\mathcal{N}(\mathcal{R})}\left(A A^{*}\right)=\lim _{n \rightarrow \infty} \operatorname{tr}_{\mathcal{N}(\mathcal{R})}\left(\chi_{X_{n}} A A^{*}\right)=\lim _{n \rightarrow \infty} \operatorname{tr}_{\mathcal{N}(\mathcal{R})}\left(A_{n} A_{n}^{*}\right)
$$

The assertion now follows from Lemma 3.2.

Lemma 3.6. If $\operatorname{det}_{\mathcal{N}(G \curvearrowright X)}(A) \geq 1$ for every $n \geq 1$ and every positive matrix $A \in M(n \times$ $\left.n ; L^{\infty}(X, \mathbb{Z}) * G\right)$, then $\operatorname{det}_{\mathcal{N}(G \curvearrowright X)}(B) \geq 1$ holds for all $m, n \geq 1$ and every matrix $B \in$ $M\left(m \times n ; L^{\infty}(X, \mathbb{Z}) * G\right)$.

Proof. This directly follows from the identity

$$
\operatorname{det}_{\mathcal{N}(G \curvearrowright X)}(B)=\operatorname{det}_{\mathcal{N}(G \curvearrowright X)}\left(B B^{*}\right)^{1 / 2} .
$$

At certain stages in the proof of Theorem 3.1 it is convenient to allow for the flexibility of non-free actions on a probability space. Let $G \curvearrowright(X, \mu)$ be a, not necessarily free, standard action. The crossed product ring $L^{\infty}(X) * G$ with its trace can be completed to a von Neumann algebra $L^{\infty}(X) *_{\mathrm{vN}} G$. This von Neumann algebra is the von Neumann algebra associated to the translation groupoid of the action [27, XIII §2]. If the action is essentially free, then $L^{\infty}(X) * G=\mathcal{N}(\mathcal{R})$ where $\mathcal{R} \subset X \times X$ is the orbit equivalence relation of $G \curvearrowright X$. On the other extreme, if $X$ is just a point, then we have $\mathcal{N}(\mathcal{R})=\mathbb{C}$ and $L^{\infty}(X) *_{\mathrm{vN}} G=\mathcal{N}(G)$.

Lemma 3.7. Assume that $G$ satisfies $M D C$. Let $G \curvearrowright X$ be a (not necessarily free) standard action. Then

$$
\operatorname{det}_{L^{\infty}(X) *_{\mathrm{vN}} G}(A) \geq 1
$$

for every $A \in M\left(m \times n, L^{\infty}(X ; \mathbb{Z}) * G\right)$.

Proof. Let $G \curvearrowright Y$ be an essentially free, standard action; take, for example, $Y=[0,1]^{G}$ with its shift action. Then the diagonal action of $G$ on the product probability space $X \times Y$ is essentially free. The projection pr : $X \times Y \rightarrow X$ induces a trace-preserving $*$-homomorphism

$$
\operatorname{pr}^{*}: L^{\infty}(X) * G \rightarrow L^{\infty}(X \times Y) * G, \quad \sum f_{g} \cdot g \mapsto \sum\left(f_{g} \circ \mathrm{pr}\right) \cdot g,
$$

which extends to the von Neumann algebras. Since $G \curvearrowright X \times Y$ satisfies MDC by hypothesis, the assertion follows (see Remark 2.13).

The remainder of this section is devoted to the proof of Theorem 3.1. Because of Lemma 3.5 it suffices in each case to show MDC only for matrices in the crossed product ring. 


\subsection{The trivial group and transition to subgroups.}

Proof of Theorem 3.1 (1). Let $(X, \mu)$ be a standard probability space. Let $A \in M(m \times$ $\left.n, L^{\infty}(X, \mathbb{Z})\right)$. We have to show that

$$
\operatorname{det}_{L^{\infty}(X)}(A) \geq 1 \text {. }
$$

By [19, Lemma 4.1] there is a unitary matrix $U \in M\left(m \times m, L^{\infty}(X)\right)$ such that $U^{-1} A A^{*} U$ is a diagonal matrix whose diagonal entries $f_{1}, f_{2}, \ldots, f_{m} \in L^{\infty}(X)$ are positive functions. We conclude from [20, Theorem 3.14 (1) and (4) and Lemma 3.15 (3), (4) and (7) on p. 128/129] that

$$
\operatorname{det}_{L^{\infty}(X)}(A)^{2}=\prod_{i=1}^{m} \operatorname{det}_{L^{\infty}(X)}\left(f_{i}\right) .
$$

According to [20, Example 3.13 on p. 128] we have

$$
\operatorname{det}_{L^{\infty}(X)}\left(f_{i}\right)=\exp \left(\int_{X} \ln \left(f_{i}(x)\right) \cdot \chi_{\left\{x \in X \mid f_{i}(x)>0\right\}} d \mu(x)\right) .
$$

Combining the aforementioned equalities yields

$$
\operatorname{det}_{L^{\infty}(X)}(A)^{2}=\exp \left(\int_{X} \ln \left(\prod_{\substack{i=1,2, \ldots, m \\ f_{i}(x)>0}} f_{i}(x)\right) d \mu(x)\right) .
$$

Fix $x \in X$. Then $A(x) A(x)^{*}$ is a matrix in $M(m \times m, \mathbb{Z})$. Let $p(t)$ be its characteristic polynomial. It can be written as $p(t)=t^{a} \cdot q(t)$ for a polynomial $q(t)$ with integer coefficients and $q(0) \neq 0$. Then $q(0)$ is the product of the positive eigenvalues of $A(x) A(x)^{*}$, i.e.

$$
\prod_{\substack{i=1,2, \ldots, m \\ f_{i}(x)>0}} f_{i}(x)=q(0)
$$

Now the assertion follows from $q(0) \geq 1$ and (3.1).

Proof of Theorem 3.1 (2). Let $i: H \rightarrow G$ be the inclusion of a subgroup, and let $(X, \mu)$ be a standard probability space endowed with an essentially free standard $H$-action. Let $i_{!} X$ be the coinduction of $X$, i.e. the $G$-space $\operatorname{map}_{H}(G, X)$, on which $g \in G$ acts from the left by composition with the $G$-map $r_{g^{-1}}: G \rightarrow G, g_{0} \mapsto g_{0} g^{-1}$. By choosing a set theoretic section $s: G / H \rightarrow G$ of the projection with $s(1)=1$, we obtain a bijection

$$
i_{!} X \stackrel{\cong}{\longrightarrow} \prod_{g H \in G / H} X, \quad \phi \mapsto(\phi(s(g H)))_{g H \in G / H} .
$$

We endow $i_{!} X$ with the structure of a standard probability space $\left(i_{1} X, \nu\right)$ by pulling back the product measure on $\prod_{g H \in G / H} X$. This structure does not depend on the choice of $s$; the measure $\nu$ is $G$-invariant $[14,3.4]$.

Let $\mathrm{pr}: i_{1} X \rightarrow X$ be the map sending $\phi$ to $\phi(1)$. We obtain a trace preserving, $H$ equivariant $*$-homomorphism $\mathrm{pr}^{*}: L^{\infty}(X) \rightarrow L^{\infty}\left(i_{!} X\right)$ by composition with pr. Thus we obtain a trace preserving $*$-algebra homomorphism

$$
u: L^{\infty}(X) * H \rightarrow L^{\infty}\left(i_{!} X\right) * G, \quad \sum_{h \in H} \lambda_{h} \cdot h \mapsto \sum_{h \in H} \operatorname{pr}^{*}\left(\lambda_{h}\right) \cdot h,
$$

which extends to the von Neumann algebras $u: \mathcal{N}(H \curvearrowright X) \rightarrow \mathcal{N}\left(G \curvearrowright i_{!} X\right)[3 ; 22$, Theorem 1.47]. 
Let $A \in M\left(m \times n ; L^{\infty}(X, \mathbb{Z}) * H\right)$. Let $u_{*} A \in M\left(m \times n ; L^{\infty}\left(i_{!} X, \mathbb{Z}\right) * G\right)$ be the matrix obtained from $A$ by applying elementwise the ring homomorphism $u$. By hypothesis, $\operatorname{det}_{\mathcal{N}\left(G \curvearrowright i_{!} X\right)}\left(u_{*} A\right) \geq 1$. The assertion follows from $\operatorname{det}_{\mathcal{N}(H \curvearrowright X)}(A)=\operatorname{det}_{\mathcal{N}(G \curvearrowright i ! X)}\left(u_{*} A\right)$ (Remark 2.13).

\subsection{Extensions with amenable quotients.}

Proof of Theorem 3.1 (3). Let $G^{\prime} \subset G$ be a subgroup. Then $H^{\prime}=H \cap G^{\prime}$ is normal in $G^{\prime}$, and $G^{\prime} / H^{\prime}$ injects into $G / H$, thus $G^{\prime} / H^{\prime}$ is also amenable. Obviously, $H^{\prime} \curvearrowright X$ satisfies MDC, if $H \curvearrowright X$ does. We have to show that $\operatorname{det}_{\mathcal{N}(G \curvearrowright X)}(A) \geq 1$ for every matrix $A$ over the ring $L^{\infty}(X) * G$. Taking $G^{\prime} \subset G$ to be the subgroup generated by the finitely many elements of $G$ appearing in such $A$, it is enough to show that $G^{\prime} \subset X$ satisfies MDC for every finitely generated subgroup $G^{\prime} \subset G$. By our first remark, we thus may and will assume that $G$ is finitely generated.

Let $p: G \rightarrow G / H$ be the projection. We choose a left-invariant word-metric $d$ on the finitely generated group $G / H$. For $R>0$ and a subset $Z \subset G / H$ we define

$$
N_{R}(Z)=\{x \in G / H \mid d(x, Z) \leq R \text { and } d(x, G / H-Z) \leq R\} .
$$

By amenability (compare [20, Lemma 13.40 on p. 469]) there exists an increasing exhaustion of $G / H$ by finite subsets $Z_{1} \subset Z_{2} \subset Z_{3} \subset \cdots \subset G / H$ (Følner exhaustion) such that for all $R>0$ and $\epsilon>0$ we find $N \in \mathbb{N}$ satisfying $\left|N_{R}\left(Z_{n}\right)\right| \leq \epsilon \cdot\left|Z_{n}\right|$ for all $n \geq N$. Let $S$ be a transversal for $H$ in $G$. We set $S_{n}=\left\{s \in S \mid s H \in Z_{n}\right\} \subset G$. We have $\left|S_{n}\right|=\left|Z_{n}\right|$. Let $p_{n}: L^{2}(G \curvearrowright X) \rightarrow L^{2}(G \curvearrowright X)$ be the projection onto the closure of the span of $p^{-1}\left(Z_{n}\right)$ and $L^{2}(X)$, i.e.

$$
p_{n}\left(\sum_{g \in G} r_{g} \cdot g\right)=\sum_{\substack{g \in G \\ g H \in Z_{n}}} r_{g} \cdot g .
$$

The map $p_{n}$ is not $L^{\infty}(X) * G$-equivariant in general but $L^{\infty}(X) * H$-equivariant.

We remark that in the group case $(X=\{*\})$ a more general statement, where $H$ is not necessarily normal, is stated in [25, Section 4] and [20, Proposition 13.93 on p. 469]. However, the proofs of these statements are not correct: the mistakes are related to the equivariance of the map $p_{n}$ above for which normality of $H$ is essential.

Notice that we have an isometric $L^{\infty}(X) * H$-equivariant isomorphism

$$
u_{n}: L^{2}(H \curvearrowright X)^{\left|S_{n}\right|} \rightarrow \operatorname{im}\left(p_{n}\right), \quad\left(f_{s}\right)_{s \in S_{n}} \mapsto \sum_{s \in S_{n}} f_{s} \cdot s .
$$

Let $A \in M\left(d \times d^{\prime} ; L^{\infty}(X, \mathbb{Z}) * G\right)$. In order to show $\operatorname{det}_{\mathcal{N}(G \curvearrowright X)}(A) \geq 1$ we may assume that $d=d^{\prime}$ and $A$ is positive (Lemma 3.6). Consider the operator

$$
L^{2}(H \curvearrowright X)^{d\left|S_{n}\right|} \stackrel{\oplus u_{n}}{\longrightarrow} \operatorname{im}\left(p_{n}\right)^{d} \stackrel{r_{A}}{\longrightarrow} L^{2}(G \curvearrowright A)^{d} \stackrel{\oplus p_{n}}{\longrightarrow} \operatorname{im}\left(p_{n}\right)^{d} \stackrel{\oplus u_{n}^{-1}}{\longrightarrow} L^{2}(H \curvearrowright X)^{d\left|S_{n}\right|} .
$$

It is easy to see that this operator is given by right multiplication with a positive matrix $A_{n} \in M\left(d \cdot\left|Z_{n}\right| \times d \cdot\left|Z_{n}\right| ; L^{\infty}(X, \mathbb{Z}) * H\right)$. By hypothesis, we have $\operatorname{det}_{\mathcal{N}(H \curvearrowright X)}\left(A_{n}\right) \geq 1$ for every $n \geq 1$. Since $\left\|p_{n}\right\| \leq 1$ holds for all $n \in \mathbb{N}$, we conclude $\left\|r_{A_{n}}\right\| \leq\left\|r_{A}\right\|$.

By Lemma 3.2 it suffices to show that

$$
\lim _{n \rightarrow \infty} \frac{\operatorname{tr}_{\mathcal{N}(H \curvearrowright X)}\left(A_{n}^{m}\right)}{d \cdot\left|Z_{n}\right|}=\frac{\operatorname{tr}_{\mathcal{N}(G \curvearrowright X)}\left(A^{m}\right)}{d} \text { for all } m \in \mathbb{N} .
$$


This is proven in the case $X=\{*\}$ in [20, Lemma 13.42 on page 470], and the proof is essentially the same in our setting.

\subsection{Extensions with finite kernels.}

Proof of Theorem 3.1 (4). Let $Q \curvearrowright X$ be an essentially free, standard action. Let $G$ act on $X$ via $p: G \rightarrow Q$. In the sequel, we write $F=l^{2}\left(L^{\infty}(X) *_{\mathrm{vN}} G\right)$ for the GNS-construction of the von Neumann algebra $L^{\infty}(X) *_{\mathrm{vN}} G$.

Let $N_{K} \in G$ be the element $\sum_{g \in K} g$. Consider the Hilbert $L^{\infty}(X) *_{\mathrm{vN}} G$-morphism

$$
r_{|K|^{-1} \cdot N_{K}}: F \rightarrow F
$$

Then $r_{|K|^{-1} \cdot N_{K}}$ is an orthogonal projection with the $K$-fixed points $F^{K}$ as image since $K \subseteq G$ is normal and $K$ acts trivially on $X$. In particular, $F^{K}$ is a finitely generated Hilbert $L^{\infty}(X) *_{\mathrm{vN}} G$-module. Define an isometric bijective operator

$$
v: L^{2}(Q \curvearrowright X) \stackrel{\cong}{\rightrightarrows} F^{K}, \sum_{q \in Q} \lambda_{q} \cdot q \mapsto|K|^{-1 / 2} \cdot \sum_{g \in G} \lambda_{p(g)} \cdot g .
$$

We also set

$$
w=v^{-1} \circ r_{|K|^{-1} \cdot N_{K}}: F \rightarrow L^{2}(Q \curvearrowright X) .
$$

For every $g \in G, f \in L^{\infty}(X)$, and $a \in L^{2}(Q \curvearrowright X)$ we have

$$
\begin{gathered}
v\left(r_{p(g)}(a)\right)=r_{g}(v(a)), \\
v\left(r_{f}(a)\right)=r_{f}(v(a)) .
\end{gathered}
$$

This, in particular, implies that the image of a $\mathcal{N}(Q \curvearrowright X)$-invariant subspace $L^{2}(Q \curvearrowright$ $X)^{d}$ under $\operatorname{diag}(v): L^{2}(Q \curvearrowright X)^{d} \rightarrow\left(F^{K}\right)^{d}$ is $L^{\infty}(X) *_{\mathrm{vN}} G$-invariant. Upon choosing an isometric embedding into $L^{2}(Q \curvearrowright X)^{d}$ and thus into $\left(F^{K}\right)^{d}$ via $\operatorname{diag}(v)$, we can equip every finitely generated Hilbert $\mathcal{N}(Q \curvearrowright X)$-module $V$ with the structure of a finitely generated Hilbert $L^{\infty}(X) *_{\mathrm{vN}} G$-module. We denote $V$ endowed with this new structure by $\operatorname{res}_{p} V$. Because of (3.2) these module structures are related by $g \phi(x)=p(g) x$ and $f \phi(x)=f x$, where we denote the identity $V \rightarrow \operatorname{res}_{p} V$ by $\phi$ for better distinction. From this we also see that the module structure on $\operatorname{res}_{p} V$ does not depend on the chosen embedding $V \hookrightarrow L^{2}(Q \curvearrowright$ $X)^{d}$ and that every morphism $f: V \rightarrow W$ of finitely generated Hilbert $\mathcal{N}(Q \curvearrowright X)$-modules induces a morphism $\operatorname{res}_{p} f: \operatorname{res}_{p} V \rightarrow \operatorname{res}_{p} W$ of finitely generated Hilbert $L^{\infty}(X) *_{\mathrm{vN}} G$ modules by the same map. Next we show that

$$
\operatorname{tr}_{L^{\infty}(X) *_{\mathrm{vN}} G}\left(\operatorname{res}_{p} f\right)=\frac{1}{|K|} \cdot \operatorname{tr}_{\mathcal{N}(Q \curvearrowright X)}(f) .
$$

It suffices to treat the case $V=L^{2}(Q \curvearrowright X)$. Let $e_{G} \in F$ and $e_{Q} \in L^{2}(Q \curvearrowright H)$ be the elements given by the unit element in the rings $L^{\infty}(X) * G$ and $L^{\infty}(X) * Q$. Write $f\left(e_{Q}\right)=\sum_{q \in Q} \lambda_{q} \cdot q$. Then

$$
w^{*} \circ f \circ w\left(e_{Q}\right)=\frac{1}{|K|} \cdot \sum_{g \in G} \lambda_{p(g)} \cdot g
$$


This implies

$$
\begin{aligned}
\operatorname{tr}_{\mathcal{N}(Q \curvearrowright X)}(f) & =\left\langle f\left(e_{Q}\right), e_{Q}\right\rangle_{L^{2}(Q \curvearrowright X)} \\
& =\left\langle 1, \lambda_{e_{Q}}\right\rangle_{L^{2}(X)} \\
& =|K| \cdot\left\langle w^{*} \circ f \circ w\left(e_{G}\right), e_{G}\right\rangle_{F} \\
& =|K| \cdot \operatorname{tr}_{L^{\infty}(X) *_{\mathrm{vN}} G}\left(\operatorname{res}_{p} f\right) .
\end{aligned}
$$

Hence (3.3) follows.

If $\left\{E_{\lambda} \mid \lambda \in \mathbb{R}\right\}$ is the spectral family of $f: V \rightarrow V$, then $\left\{\operatorname{res}_{p} E_{\lambda} \mid \lambda \in \mathbb{R}\right\}$ is the spectral family of $\operatorname{res}_{p} f: \operatorname{res}_{p} V \rightarrow \operatorname{res}_{p} V$. Hence (3.3) successively yields that

$$
\begin{aligned}
F\left(\operatorname{res}_{p} f\right) & =\frac{1}{|K|} \cdot F(f), \\
\operatorname{det}_{L^{\infty}(X) *_{\mathrm{vN}} G}\left(\operatorname{res}_{p} f\right) & =\left(\operatorname{det}_{\mathcal{N}(Q \curvearrowright X)}(f)\right)^{1 /|K|} .
\end{aligned}
$$

Let $A \in M\left(d^{\prime} \times d, L^{\infty}(X, \mathbb{Z}) * Q\right)$. We have to show that $\operatorname{det}_{\mathcal{N}(Q \curvearrowright X)}(A) \geq 1$. By Lemma 3.6 we may and will assume that $d^{\prime}=d$ and $A$ is positive.

Let $n \in \mathbb{N}$. We get a morphism $\operatorname{res}_{p} r_{A^{n}}: \operatorname{res}_{p} L^{2}(Q \curvearrowright X)^{d} \rightarrow \operatorname{res}_{p} L^{2}(Q \curvearrowright X)^{d}$ of finitely generated Hilbert $L^{\infty}(X) *_{\mathrm{vN}} G$-modules. We have the orthogonal sum decomposition

$$
F=\underbrace{\operatorname{im}\left(r_{|K|^{-1} \cdot N_{K}}\right)}_{=F^{K}} \oplus \operatorname{im}\left(r_{1-|K|^{-1} \cdot N_{K}}\right) .
$$

Consider the morphism

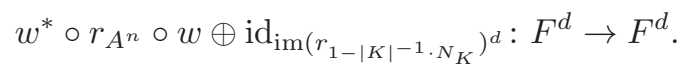

We conclude from (3.4) and [20, Theorem 3.14 (1) on p. 128 and Lemma 3.15 (7) on p. 130] that

$$
\begin{aligned}
\operatorname{det}_{\mathcal{N}(Q \curvearrowright X)}\left(r_{A}\right)^{n} & =\operatorname{det}_{\mathcal{N}(Q \curvearrowright X)}\left(r_{A^{n}}\right) \\
& =\operatorname{det}_{L^{\infty}(X) *_{\mathrm{vN}} G}\left(\operatorname{res}_{p} r_{A^{n}}\right)^{|K|} \\
& =\operatorname{det}_{L^{\infty}(X) *_{\mathrm{vN}} G}\left(w^{*} \circ r_{A^{n}} \circ w\right)^{|K|} \cdot \operatorname{det}_{L^{\infty}(X) *_{\mathrm{vN}} G}\left(\operatorname{id}_{\mathrm{im}\left(\left.r_{1-|K|}\right|_{N_{K}}\right)^{d}}\right)^{|K|} \\
& =\operatorname{det}_{L^{\infty}(X) *_{\mathrm{vN}} G}\left(w^{*} \circ r_{A^{n}} \circ w \oplus \operatorname{id}_{i m\left(r_{1-|K|-1 \cdot N_{K}}\right)^{d}}\right)^{|K|} .
\end{aligned}
$$

For $u=\sum_{q \in Q} \lambda_{q} \cdot q$ in $L^{\infty}(X, \mathbb{Z}) * Q$ let $s(u) \in L^{\infty}(X, \mathbb{Z}) * G$ be the element $\sum_{g \in G} \lambda_{p(g)} \cdot g$. Define $B=\left(b_{i, j}\right)_{i, j} \in M\left(d \times d, L^{\infty}(X, \mathbb{Z}) * G\right)$ to be the matrix obtained from $A^{n}=\left(a_{i, j}\right)$ by setting $b_{i, i}=s\left(a_{i, i}-1\right)$ and $b_{i, j}=s\left(a_{i, j}\right)$ if $i \neq j$. One easily verifies that

$$
\left.\frac{1}{|K|} \cdot r_{|K| \cdot I_{d}+B}=w^{*} \circ r_{A^{n}} \circ w \oplus \operatorname{id}_{\operatorname{im}\left(r_{1-|K|}-1 \cdot N_{K}\right.}\right)^{d},
$$

where $I_{d}$ is the identity matrix in $M\left(d \times d, L^{\infty}(X, \mathbb{Z}) * G\right)$. Notice that $|K| \cdot I_{d}+B$ lies in $M\left(d \times d, L^{\infty}(X, \mathbb{Z}) * G\right)$; thus, by hypothesis and Lemma 3.7, $\operatorname{det}_{L^{\infty}(X) *_{\mathrm{vN}} G}\left(r_{|K| \cdot I_{d}+B}\right) \geq 1$. 
We conclude from [20, Theorem 3.14 (1) on page 128] that

$$
\begin{aligned}
\operatorname{det}_{L^{\infty}(X) *_{\mathrm{vN}} G}\left(w^{*} \circ r_{A^{n}} \circ w \oplus \operatorname{id}_{\mathrm{im}\left(r_{1-|K|^{-1, N_{K}}}\right)^{d}}\right) & =\operatorname{det}_{L^{\infty}(X) *_{\mathrm{vN}} G}\left(\frac{1}{|K|} \cdot r_{|K| \cdot I_{d}+B}\right) \\
& =\frac{1}{|K|^{d}} \cdot \operatorname{det}_{L^{\infty}(X) *_{\mathrm{vN}} G}\left(r_{|K| \cdot I_{d}+B}\right) \\
& \geq \frac{1}{|K|^{d}} .
\end{aligned}
$$

We conclude from (3.5) and (3.6) that

$$
\operatorname{det}_{\mathcal{N}(Q \curvearrowright X)}\left(r_{A}\right) \geq|K|^{-d|K| / n}
$$

holds for every $n \in \mathbb{N}$. Hence $\operatorname{det}_{\mathcal{N}(Q \curvearrowright X)}\left(r_{A}\right) \geq 1$.

3.6. Colimits. Throughout this subsection, we consider a directed system of groups $\left\{G_{i} \mid\right.$ $i \in I\}$ over the directed set $I$. Denote its colimit by $G=\operatorname{colim}_{i \in I} G_{i}$. Let $\psi_{i}: G_{i} \rightarrow G$ for $i \in I$ and $\psi_{i, j}: G_{i} \rightarrow G_{j}$ for $i, j \in I, i \leq j$, be the structure maps. We do not require that $\psi_{i}$ or $\psi_{i, j}$ are injective.

Proof of Theorem 3.1 (5). Let $G \curvearrowright(X, \mu)$ be an essentially free, standard action. Every $G_{i}$ acts on $X$ via $\psi_{i}$ (but not necessarily free). We obtain a trace-preserving ring *-homomorphism

$$
\alpha_{i}: L^{\infty}(X) * G_{i} \rightarrow L^{\infty}(X) * G, \sum_{h \in G_{i}} l_{h} \cdot h \mapsto \sum_{h \in G_{i}} l_{h} \cdot \psi_{i}(h) .
$$

Let $A \in M\left(m \times n, L^{\infty}(X ; \mathbb{Z}) * G\right)$. Write $A=\sum_{g \in G} f_{g} g$ with $f_{g} \in M\left(m \times n, L^{\infty}(X, \mathbb{Z})\right)$. Let $i_{0} \in I$ be such that for every $i \geq i_{0}$ the implication

$$
f_{g} \neq 0 \Rightarrow g \in \operatorname{im}\left(\psi_{i}\right)
$$

holds. Let $V=\left\{g \in G \mid f_{g} \neq 0\right\}$. For every $g \in V$ let $g^{(i)} \in G_{i}$ be a preimage of $g$. Let $A_{i}=\sum_{g \in V} f_{g} \cdot g^{(i)}$ and $\Delta_{i}=A_{i} A_{i}^{*}$. We have $\alpha_{i}\left(A_{i}\right)=A$. From Lemma 3.4 it is clear that there is a uniform bound of the operator norms of the $\Delta_{i} \in M\left(n \times n, L^{\infty}(X) *_{\mathrm{vN}} G_{i}\right)$. By hypothesis and Lemma 3.7 we have

$$
\operatorname{det}_{L^{\infty}(X) *_{\mathrm{vN}} G_{i}}\left(A_{i}\right) \geq 1 \text {. }
$$

Let $m \geq 1$. The assertion would follow from Lemma 3.2 provided we show that

$$
\operatorname{tr}_{\mathcal{N}(G \curvearrowright X)}\left(\Delta^{m}\right)=\lim _{i \in I} \operatorname{tr}_{L^{\infty}(X) *_{\mathrm{vN}} G_{i}}\left(\Delta_{i}^{m}\right) .
$$

We can find $i_{1} \geq i_{0}$ in $I$ such that for every $h \in G_{i_{1}}$ with $l_{h} \neq 0 \in M\left(n \times n, L^{\infty}\left(X_{i_{1}} \times X\right)\right)$ in the finite linear combination $\Delta_{i_{1}}^{m}=\sum_{h} l_{h} h$ we have the implication

$$
\psi_{i_{1}}(h)=1 \Rightarrow h=1 .
$$

For $i \geq i_{1}$ the right hand side of (3.7) is stationary and equal to the left hand side. 


\subsection{Bernoulli actions.}

Proof of Theorem 3.1 (6). For any countable set $A$ denote by $\mu_{A}$ the product measure $\bigotimes_{a \in A} \mu$ on $X^{A}$. The $\sigma$-algebra of Borel sets in $X^{G}=\prod_{g \in G} X$ is the $\sigma$-algebra $\mathcal{S}$ generated by

$$
\mathcal{B}=\left\{\prod_{g \in G} U_{g} \mid U_{g}=X \text { for almost all } g \in G\right\} .
$$

Let $\mathcal{A}$ be the algebra generated by $\mathcal{B}$. We say that a measurable function $f: X \rightarrow \mathbb{Z}$ is $\mathcal{A}$ measurable if $f^{-1}(z) \in \mathcal{A}$ for every $z \in \mathbb{Z}$. Any set $M \in \mathcal{A}$ can be written as $M=\cup_{k=1}^{n} M_{k}$ with disjoint sets $M_{k} \in \mathcal{B}$. The sets $M \in \mathcal{A}$ have the property that there exists a finite subset $F \subseteq G$ with $M=\operatorname{pr}_{F}^{-1}\left(\operatorname{pr}_{F}(M)\right)$ where $\operatorname{pr}_{F}: \prod_{g \in G} X \rightarrow \prod_{g \in F} X$ is the projection onto the components of $F$. We denote with $F(M) \subseteq G$ the smallest subset with this property. Let $R$ be the ring of all bounded, $\mathcal{A}$-measurable functions $X^{G} \rightarrow \mathbb{Z}$. For $f \in R$, we denote with $F(f) \subset G$ the (finite) union $F(f)=\bigcup_{z \in \mathbb{Z}} F\left(f^{-1}(z)\right.$ ). Since any non-empty set in $\mathcal{A}$ has positive measure, the ring $R$ embeds into $L^{\infty}\left(X^{G}, \mathbb{Z}\right)$. Since $R$ is $G$-invariant, we obtain a subring $R * G \subset L^{\infty}\left(X^{G}, \mathbb{Z}\right) * G$ that is closed under the involution.

Let $\psi_{i}: G \rightarrow G_{i}, i \in I$, be the structure maps of the limit. The map $\psi_{i}$ induces a measurable map

$$
\alpha_{i}: X^{G_{i}} \rightarrow X^{G}, \alpha_{i}\left(\left(x_{h}\right)\right)_{g}=x_{\psi_{i}(g)} .
$$

If $\left.\psi_{i}\right|_{F(M)}$ is injective for $M \in \mathcal{A}$, then $\mu_{G_{i}}\left(\alpha_{i}^{-1}(M)\right)=\mu_{G}(M)$. Since every $f \in R$ is a finite linear combinations of characteristic functions of sets in $\mathcal{A}$, we also obtain that

$$
\int_{X^{G_{i}}} f \circ \alpha_{i}(z) d \mu_{G_{i}}(z)=\int_{X^{G}} f(x) d \mu_{G}(x)
$$

provided $\left.\psi_{i}\right|_{F(f)}$ is injective.

We obtain a ring homomorphism which respects the involutions:

$$
\beta_{i}: R * G \rightarrow L^{\infty}\left(X^{G_{i}}\right) * G_{i}, \sum_{g \in G} f_{g} \cdot g \mapsto \sum_{g \in G}\left(f_{g} \circ \alpha_{i}\right) \cdot \psi_{i}(g) .
$$

By applying this homomorphism entry-wise we obtain a ring homomorphism $M\left(d \times d^{\prime}, R *\right.$ $G) \rightarrow M\left(d \times d^{\prime}, L^{\infty}\left(X^{G_{i}}\right) * G_{i}\right)$ that we denote by the same name.

Lemma 3.8. Let $A \in M\left(d \times d^{\prime}, R * G\right)$ and $\Delta=A A^{*}$. Let $\Delta_{i}=\beta_{i}(A) \beta_{i}(A)^{*}$. Let $m \geq 1$. Then

$$
\operatorname{tr}_{\mathcal{N}\left(G \curvearrowright X^{G}\right)}\left(\Delta^{m}\right)=\lim _{i \rightarrow \infty} \operatorname{tr}_{\mathcal{N}\left(G_{i} \curvearrowright X^{\left.G_{i}\right)}\right.}\left(\Delta_{i}^{m}\right),
$$

and we have $\operatorname{det}_{\mathcal{N}\left(G \curvearrowright X^{G}\right)}(A) \geq 1$.

Proof of lemma. By considering the matrix entries separately, the assertion reduces to showing that for any set of $2 m$ elements $a_{1}, \ldots, a_{m}, b_{1}, \ldots, b_{m} \in R * G$ we have

$$
\operatorname{tr}_{\mathcal{N}\left(G \curvearrowright X^{G}\right)}\left(a_{1} b_{1}^{*} a_{2} b_{2}^{*} \cdots a_{m} b_{m}^{*}\right)=\lim _{i \rightarrow \infty} \operatorname{tr}_{\mathcal{N}\left(G_{i} \curvearrowright X^{G_{i}}\right)}\left(\beta_{i}\left(a_{1}\right) \beta_{i}\left(b_{1}\right)^{*} \cdots \beta_{i}\left(a_{m}\right) \beta_{i}\left(b_{m}\right)^{*}\right) .
$$

Since $\beta_{i}$ is a ring $*$-homomorphism, the assertion reduces further to showing that for $f \in R$ and $g \in G$ we have

$$
\operatorname{tr}_{\mathcal{N}\left(G \curvearrowright X^{G}\right)}(f \cdot g)=\lim _{i \rightarrow \infty} \operatorname{tr}_{\mathcal{N}\left(G_{i} \curvearrowright X^{G_{i}}\right)}\left(\beta_{i}(f \cdot g)\right) .
$$

Choose $i_{0} \in I$ such that $\left.\psi_{i}\right|_{F(f) \cup\{1, g\}}$ is injective for $i \geq i_{0}$. Then (3.8) yields that

$$
\operatorname{tr}_{\mathcal{N}\left(G \curvearrowright X^{G}\right)}(f \cdot g)=\operatorname{tr}_{\mathcal{N}\left(G_{i} \curvearrowright X^{G_{i}}\right)}\left(\beta_{i}(f \cdot g)\right)
$$


for $i \geq i_{0}$. This concludes the proof of (3.9). By Lemma 3.4 there is an upper bound $\left\|r_{\Delta_{i}^{m}}\right\|$ that is independent of $i$. By hypothesis, $\operatorname{det}_{\mathcal{N}\left(G_{i} \curvearrowright X^{G_{i}}\right)}\left(\beta_{i}(A)^{m}\right) \geq 1$. Finally, Lemma 3.2 implies that $\operatorname{det}_{\mathcal{N}\left(G \curvearrowright X^{G}\right)}(A) \geq 1$.

We need a general fact before we can complete the proof: Let $f_{1}, f_{2} \ldots, f_{m} \in L^{\infty}(X)$. For every $1 \leq i \leq m$ let $f_{i}^{(n)} \in L^{\infty}(X)$ be a sequence such that there is a constant $C>0$ with $\left\|f_{i}^{(n)}\right\|_{L^{\infty}(X)}<C$ and $\lim _{n \rightarrow \infty}\left\|f_{i}-f_{i}^{(n)}\right\|_{L^{1}(X)}=0$. Then:

$$
\lim _{n \rightarrow \infty}\left\|f_{1} \cdots f_{m}-f_{1}^{(n)} \cdots f_{m}^{(n)}\right\|_{L^{1}(X)}=0 .
$$

This follows from an iterated application of the corresponding assertion for $m=2$. For $m=2$ we have:

$$
\begin{aligned}
& \int_{X}\left|f_{1}(x) f_{2}(x)-f_{1}^{(n)}(x) f_{2}^{(n)}(x)\right| d \mu(x) \\
& \quad \leq\left\|f_{2}\right\|_{L^{\infty}(X)} \int_{X}\left|f_{1}(x)-f_{1}^{(n)}(x)\right| d \mu(x)+\left\|f_{1}^{(n)}\right\|_{L^{\infty}(X)} \int_{X}\left|f_{2}(x)-f_{2}^{(n)}(x)\right| d \mu(x) \\
& \quad \leq\left\|f_{2}\right\|_{L^{\infty}(X)} \cdot\left\|f_{1}-f_{1}^{(n)}\right\|_{L^{1}(X)}+C \cdot\left\|f_{2}-f_{2}^{(n)}\right\|_{L^{1}(X)} \stackrel{n \rightarrow \infty}{\longrightarrow} 0 .
\end{aligned}
$$

Now we can complete the proof of Theorem 3.1 (6). For any $S \in \mathcal{S}$ and any $\epsilon>0$ there exists a set $M \in \mathcal{A}$ with $\mu_{G}(S \triangle M)<\epsilon$. Since every element in $f \in L^{\infty}(X, \mathbb{Z})$ is a finite linear combination of characteristic functions, there exist functions $f^{(n)} \in R$ associated to $f$ such that $\left\|f^{(n)}\right\|_{L^{\infty}(X)} \leq\|f\|_{L^{\infty}(X)}$ for all $n \geq 1$ and $\left\|f-f^{(n)}\right\|_{L^{1}(X)}<1 / n$. Let $A \in M\left(d \times d^{\prime}, L^{\infty}(X) * G\right)$. Let $B^{(n)} \in M\left(d \times d^{\prime}, R * G\right)$ be the matrix obtained from $A$ by replacing each entry $\sum f_{g} \cdot g$ with $\sum f_{g}^{(n)} \cdot g$. Let $\Delta=A A^{*}$ and $\Delta_{n}=B^{(n)}\left(B^{(n)}\right)^{*}$. For every $m \geq 1$ we have

$$
\operatorname{tr}_{\mathcal{N}(G \curvearrowright X)}\left(\Delta^{m}\right)=\lim _{n \rightarrow \infty} \operatorname{tr}_{\mathcal{N}(G \curvearrowright X)}\left(\Delta_{n}^{m}\right)
$$

By considering matrix entries separately as in the proof of Lemma 3.8, this easily follows from (3.10) and the $G$-invariance of the measure. By Lemma 3.4, $\left\|r_{\Delta_{n}^{m}}\right\|$ has an upper bound independent of $n$. Lemma 3.8 and Lemma 3.2 complete the proof.

\section{Proofs of Theorems 1.9 And 1.10}

The following two results are proved in first author's book [20] for group von Neumann algebras. We need them for arbitrary finite von Neumann algebras; the proof translates literally to the general case.

Proposition 4.1 ([20, Theorem 3.35 (5) on p. 142]). Let $\mathcal{A}$ be a finite von Neumann algebra with a fixed trace. Let $\phi_{*}: C_{*} \rightarrow D_{*}$ be a chain map of finitely generated Hilbert $\mathcal{A}$-chain complexes. Suppose that $b_{n}^{(2)}\left(C_{*}\right)=b_{n}^{(2)}\left(D_{*}\right)=0$ and $\operatorname{det}_{\mathcal{A}}\left(c_{n}\right)$, $\operatorname{det}_{\mathcal{A}}\left(d_{n}\right)>0$ for all $n \in \mathbb{Z}$. Then the mapping cone cone $_{*}\left(\phi_{*}\right)$ is also a finitely generated Hilbert $\mathcal{A}$-chain complex with vanishing $L^{2}$-Betti numbers and positive determinants of his differentials. Moreover, we obtain the equation

$$
\rho^{(2)}\left(\operatorname{cone}_{*}\left(\phi_{*}\right)\right)=\rho^{(2)}\left(D_{*}\right)-\rho^{(2)}\left(C_{*}\right) .
$$


Lemma 4.2 ([20, Lemma 3.41 on p. 146]). Let $C_{*}$ be a finitely generated Hilbert $\mathcal{A}$-chain complexes and $\gamma_{*}$ a chain contraction. Then $b_{n}^{(2)}\left(C_{*}\right)=0$ for all $n \in \mathbb{Z}$. If $\operatorname{det}_{\mathcal{A}}\left(c_{n}\right)>0$ for all $n \in \mathbb{Z}$ then

$$
\rho^{(2)}\left(C_{*}\right)=\ln \operatorname{det}_{\mathcal{A}}\left(\left(c_{*}+\gamma_{*}\right)_{\text {odd }}: \oplus_{n \in \mathbb{Z}} C_{2 n+1} \rightarrow \oplus_{n \in \mathbb{Z}} C_{2 n}\right) .
$$

Definition 4.3. Let $R$ be a ring with involution. A finitely generated based free $R$-module is a finitely generated free $R$-module together with an isomorphism $M \cong R^{n}$. A finitely generated based projective $R$-module $P$ is a finitely generated projective $R$-module $P$ together with an isomorphism $P \cong R^{n} A$ where $A \in M(n \times n, R)$ satisfies $A^{2}=A$ and $A^{*}=A$.

Remark 4.4. Consider a standard action $G \curvearrowright X$ and a measurable subset $A \subset X$ such that there is a finite subset $S \subset G$ with $S \cdot A=X$ up to null sets. We can find (and fix for the following discussion) measurable subsets $A_{g} \subset A$ for each $g \in S$ such that the sets $g A_{g}$ partition $X$. We obtain the isomorphism of left $\chi_{A}\left(L^{\infty}(X, \mathbb{Z}) * G\right) \chi_{A}$-modules

$$
\phi: \chi_{A}\left(L^{\infty}(X, \mathbb{Z}) * G\right) \stackrel{\cong}{\longrightarrow} \bigoplus_{g \in S} \chi_{A}\left(L^{\infty}(X, \mathbb{Z}) * G\right) \chi_{A_{g}}, \phi(x)=\sum_{g \in S} x g \chi_{A_{g}} .
$$

If $\psi: F \stackrel{\cong}{\longrightarrow}\left(L^{\infty}(X, \mathbb{Z}) * G\right)^{n}$ is a finitely generated based free module, then $\chi_{A} F$ becomes a finitely generated based projective module over the $\operatorname{ring} \chi_{A} L^{\infty}(X, \mathbb{Z}) * G \chi_{A}$ by

$$
\chi_{A} F \stackrel{\chi_{A} \psi}{\longrightarrow}\left(\chi_{A} L^{\infty}(X, \mathbb{Z}) * G\right)^{n} \stackrel{\oplus_{i=1}^{n} \oplus_{S} \phi}{\longrightarrow}\left(\chi_{A} L^{\infty}(X, \mathbb{Z}) * G \chi_{A}\right)^{|S| n} Q,
$$

where is $Q$ is the projection matrix

$$
Q=\operatorname{diag}\left(\left(\chi_{A_{g}}\right)_{g \in S}\right) \oplus \cdots \oplus \operatorname{diag}\left(\left(\chi_{A_{g}}\right)_{g \in S}\right) .
$$

Let $P \cong\left(\chi_{A} L^{\infty}(X, \mathbb{Z}) * G \chi_{A}\right)^{n} Q^{\prime}$ be a finitely generated based projective $\chi_{A} L^{\infty}(X, \mathbb{Z}) *$ $G \chi_{A}$-module. Then $L^{2}(G \curvearrowright X \mid A) \otimes_{\chi_{A} L^{\infty}(X, \mathbb{Z}) * G \chi_{A}} P$ is isomorphic to the image of the orthogonal projection

$$
L^{2}\left(\left.G \curvearrowright X\right|_{A}\right)^{n} \rightarrow L^{2}\left(\left.G \curvearrowright X\right|_{A}\right)^{n}, x \mapsto x Q^{\prime},
$$

and obtains the structure of a Hilbert $\mathcal{N}(G \curvearrowright X \mid A)$-module from this isomorphism.

Let $F$ be a finitely generated based free module over the $\operatorname{ring} L^{\infty}(X, \mathbb{Z}) * G$. Since $\chi_{A}$ is full, there is an obvious isomorphism

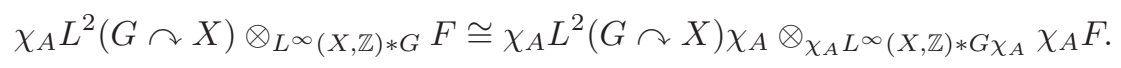

If $V$ is any Hilbert $\mathcal{N}(G \curvearrowright X)$-module, then $\chi_{A} V$ becomes a Hilbert $\mathcal{N}\left(\left.G \curvearrowright X\right|_{A}\right)$-module (see [16, pp. 19-27]). Since $L^{2}(G \curvearrowright X) \otimes_{L^{\infty}(X, \mathbb{Z}) * G} F$ is a Hilbert $\mathcal{N}(G \curvearrowright X)$-module through the free basis of $F$, the left hand side in (4.2) is a Hilbert $\mathcal{N}\left(\left.G \curvearrowright X\right|_{A}\right)$-module. On the other hand, the right hand side in (4.2) is a Hilbert $\mathcal{N}\left(\left.G \curvearrowright X\right|_{A}\right)$-module since $\chi_{A} F$ is based projective. The isomorphism (4.2) is an isomorphism of Hilbert $\mathcal{N}\left(\left.G \curvearrowright X\right|_{A}\right)$ modules.

Lemma 4.5. Let $G \curvearrowright(X, \mu)$ be an essentially free, standard action and $A \subset X$ a measurable subset such there is a finite subset $F \subset G$ with $F A=X$ up to null sets. Then the following statements are equivalent:

(1) $G \curvearrowright X$ satisfies $M D C$.

(2) For every homomorphism of finitely generated based projective $L^{\infty}(X, \mathbb{Z}) * G$-modules $f: P \rightarrow Q$ the associated Hilbert $\mathcal{N}(G \curvearrowright X)$-morphism $L^{2}(G \curvearrowright X) \otimes_{L^{\infty}(X, \mathbb{Z}) * G} f$ has determinant $\geq 1$. 
(3) For every homomorphism of finitely generated based projective $\chi_{A} L^{\infty}(X, \mathbb{Z}) * G \chi_{A^{-}}$ modules $f: P \rightarrow Q$ the associated Hilbert $\mathcal{N}\left(\left.G \curvearrowright X\right|_{A}\right)$-morphism

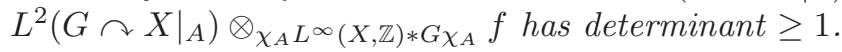

Proof. (1) implies (2): Every homomorphism $f: P \rightarrow Q$ between finitely generated based projective $L^{\infty}(X, \mathbb{Z}) * G$-modules can be extended by zero to an homomorphism

$$
P \oplus P^{\prime} \stackrel{f \oplus 0}{\longrightarrow} Q \oplus Q^{\prime}
$$

of finitely generated based free modules. We have $\operatorname{det}_{\mathcal{N}(G \curvearrowright X)}(f)=\operatorname{det}_{\mathcal{N}(G \curvearrowright X)}(f \oplus 0)$, and the latter is $\geq 1$ since $G$ satisfies MDC.

(2) implies (3): By a similar argument, it is enough to show that, for every homomorphism of finitely generated based free $\chi_{A} L^{\infty}(X, \mathbb{Z}) * G \chi_{A}$-modules $f: P \rightarrow Q$, the associated Hilbert $\mathcal{N}\left(\left.G \curvearrowright X\right|_{A}\right)$-morphism $L^{2}\left(\left.G \curvearrowright X\right|_{A}\right) \otimes_{\chi_{A} L^{\infty}(X, \mathbb{Z}) * G \chi_{A}} f$ has determinant $\geq 1$. This is equivalent to: For every matrix $B \in M\left(m \times n, \chi_{A} L^{\infty}(X, \mathbb{Z}) * G \chi_{A}\right)$ we $\operatorname{have~} \operatorname{det}_{\mathcal{N}(\mathcal{R})}(B) \geq 1$. We can view such $B$ also as an element in $M\left(m \times n, L^{\infty}(X, \mathbb{Z}) * G\right)$. It is obvious (just check on polynomials) that the spectral density function of $B$ with respect to $\mathcal{N}(G \curvearrowright X)$ is just $\mu(A)$ times the spectral density function with respect to $\mathcal{N}\left(\left.G \curvearrowright X\right|_{A}\right)$. Assuming (2), we now see that $\operatorname{det}_{\mathcal{N}\left(\left.G \curvearrowright X\right|_{A}\right)}(B) \geq 1$ and

$$
\ln \operatorname{det}_{\mathcal{N}(G \curvearrowright X)}(B)=\mu(A) \cdot \ln \operatorname{det}_{\mathcal{N}\left(\left.G \curvearrowright X\right|_{A}\right)}(B) .
$$

(3) implies (1): Let $f: F \rightarrow F^{\prime}$ be a homomorphism of finitely generated based free $L^{\infty}(X, \mathbb{Z}) * G$-modules. Since $\chi_{A}$ is full by Lemma $2.8, \chi_{A} F$ and $\chi_{A} F^{\prime}$ are finitely generated (based) projective $\chi_{A} L^{\infty}(X, \mathbb{Z}) * G \chi_{A}$-modules. By (3) and Remark 4.4 the map

$$
\chi_{A} L^{2}(G \curvearrowright X) \otimes f: \chi_{A} L^{2}(G \curvearrowright X) \otimes_{L^{\infty}(X, \mathbb{Z}) * G} F \rightarrow \chi_{A} L^{2}(G \curvearrowright X) \otimes_{L^{\infty}(X, \mathbb{Z}) * G} F^{\prime}
$$

has determinant $\geq 1$. Thus, by Remark 2.14, the map $L^{2}(G \curvearrowright X) \otimes f$ has determinant $\geq 1$. By Lemma 3.5 this suffices to show that $G \curvearrowright X$ satisfies MDC.

We omit the proof of the following lemma which is essentially the same as that of Lemma 4.5.

Lemma 4.6. Let $\mathcal{R}$ be a standard equivalence relation on $(X, \mu)$. Let $A \subset X$ be a measurable subset such that $\chi_{A}$ is a full idempotent in $\mathbb{Z R}$. Then the following statements are equivalent:

(1) $\mathcal{R}$ satisfies $M D C$.

(2) For every homomorphism of finitely generated based projective $\mathbb{Z} \mathcal{R}$-modules $f: P \rightarrow$ $Q$ the associated Hilbert $\mathcal{N}(\mathcal{R})$-morphism $L^{2}(\mathcal{R}) \otimes_{\mathbb{Z} \mathcal{R}} f$ has determinant $\geq 1$.

(3) $\left.\mathcal{R}\right|_{A}$ satisfies $M D C$.

(4) For every homomorphism of finitely generated based projective $\left.\mathbb{Z} \mathcal{R}\right|_{A}$-modules $f: P \rightarrow$ $Q$ the associated Hilbert $\mathcal{N}(\mathcal{R})$-morphism $L^{2}(\mathcal{R}) \otimes_{\mathbb{Z} \mathcal{R} \mid{ }_{A}} f$ has determinant $\geq 1$.

Proof of Theorem 1.9. By Theorem 2.3 there exist essentially free, standard actions $G \curvearrowright$ $\left(X, \mu_{X}\right)$ and $H \curvearrowright\left(Y, \mu_{Y}\right)$ that are weakly orbit equivalent. Let $f: B \rightarrow A$ be a weak orbit equivalence between measurable subsets $A \subset X$ and $B \subset Y$. By [10, Lemma 2.2] we may assume that both actions are ergodic. The map $f$ induces an isomorphism between the restricted orbit equivalence relations $\left.\mathcal{R}(G \curvearrowright X)\right|_{A}$ and $\left.\mathcal{R}(H \curvearrowright Y)\right|_{B}$. By hypothesis, $\mathcal{R}(G \curvearrowright X)$ satisfies MCD. That $\mathcal{R}(H \curvearrowright Y)$ satisfies MDC, thus $H$ satisfies the determinant conjecture, follows by applying Lemma 4.6 twice provided that $\chi_{A} \in \mathbb{Z} \mathcal{R}(G \curvearrowright X)$ and $\chi_{B} \in \mathbb{Z} \mathcal{R}(H \curvearrowright Y)$ are full idempotents. For that, we prove in general that $\chi_{A} \in \mathbb{Z} \mathcal{R}$ is a full idempotent if $\mathcal{R}$ is an ergodic standard equivalence relation and $A \subset X$ is a measurable 
subset of positive measure: Let $X=\bigcup_{i=1}^{n+1} A_{i}$ be a Borel partition of $X$ such that $A_{1}, \ldots, A_{n}$ all have the same measure as $A$ and $\mu\left(A_{n+1}\right) \leq \mu\left(A_{n}\right)$. By ergodicity and [12, Lemma 2.1] there are measure isomorphisms $\phi_{i}: X \rightarrow X$ for each $1 \leq i \leq n+1$ such that $\left(x, \phi_{i}(x)\right) \in \mathcal{R}$ for $x \in X$ and $\phi_{i}(A)=A_{i}$ for $1 \leq i<n$ and $A_{n+1} \subset \phi_{n+1}(A)$. The characteristic function $\chi_{\phi_{i}}$ of the graph of each $\phi_{i}$ is an element in $\mathbb{Z} \mathcal{R}$. From the properties of $\phi_{i}$ we obtain that

$$
1=\chi_{X}=\sum_{i=1}^{n} \chi_{\phi_{i}}^{*} \chi_{A} \chi_{\phi_{i}}+\chi_{\phi_{n+1}}^{*} \chi_{A} \chi_{\phi_{n+1}} \chi_{\mathrm{im}\left(\phi_{n+1}\right)} .
$$

Thus, $\chi_{A}$ is a full idempotent.

Proof of Theorem 1.10. Let $Z_{G}$ and $Z_{H}$ be finite CW-models of the classifying spaces $B G$ and $B H$, respectively. By Theorem 2.3 there exist essentially free, standard actions $G \curvearrowright$ $\left(X, \mu_{X}\right)$ and $H \curvearrowright\left(Y, \mu_{Y}\right)$ that are uniformly weakly orbit equivalent. Let $f: B \rightarrow A$ be a uniform weak orbit equivalence between measurable subsets $A \subset X$ and $B \subset Y$. It induces a trace-preserving ring isomorphism $f_{*}: \chi_{A} L^{\infty}(X, \mathbb{Z}) * G \chi_{A} \rightarrow \chi_{B} L^{\infty}(Y, \mathbb{Z}) * H \chi_{B}$ (Lemma 2.9) which extends to an isometry $f_{*}: L^{2}\left(\left.G \curvearrowright X\right|_{A}\right) \rightarrow L^{2}\left(\left.H \curvearrowright Y\right|_{B}\right)$. By assumption, $G$ satisfies MDC. Because of the previous ring isomorphism and by applying Lemma 4.5 twice it follows that $H \curvearrowright Y$ satisfies MDC; in particular, $H$ satisfies the determinant conjecture.

The cellular chain complex $C_{*}^{\text {cell }}\left(\tilde{Z}_{G}\right)$ of the universal cover $\tilde{Z}_{G}$ is a finite based free $\mathbb{Z} G$ resolution of $\mathbb{Z}$. By successively applying (2.1) and (2.2) to the differentials of $l^{2}(G) \otimes_{\mathbb{Z} G}$ $C_{*}^{\text {cell }}\left(\tilde{Z}_{G}\right)$, we obtain that

$$
\rho^{(2)}\left(\chi_{A} L^{2}(G \curvearrowright X) \otimes_{\mathbb{Z} G} C_{*}^{\text {cell }}\left(\tilde{Z}_{G}\right)\right)=\frac{\rho^{(2)}(G)}{\mu_{X}(A)} .
$$

The $\chi_{A} L^{\infty}(X, \mathbb{Z}) * G \chi_{A^{-}}$chain complex

$$
C(G)_{*}:=\chi_{A} L^{\infty}(X, \mathbb{Z}) * G \otimes_{\mathbb{Z} G} C_{*}^{\text {cell }}\left(\tilde{Z}_{G}\right)
$$

is a finite resolution by based projective modules of $\chi_{A} L^{\infty}(X, \mathbb{Z})=L^{\infty}(A, \mathbb{Z})$ by Lemma 2.6 and Lemma 2.8. Furthermore, we define:

$$
C(G)_{*}^{(2)}:=L^{2}\left(\left.G \curvearrowright X\right|_{A}\right) \otimes_{\chi_{A} L^{\infty}(X, \mathbb{Z}) * G \chi_{A}} C(G)_{*} .
$$

Since $\chi_{A}$ is full (Lemma 2.8),

$$
C(G)_{*}^{(2)} \cong \chi_{A} L^{2}(G \curvearrowright X) \otimes_{\mathbb{Z} G} C_{*}^{\text {cell }}\left(\tilde{Z}_{G}\right) .
$$

Hence,

$$
\rho^{(2)}\left(C(G)_{*}^{(2)}\right)=\frac{\rho^{(2)}(G)}{\mu_{X}(A)} .
$$

By replacing $G \curvearrowright X$ by $H \curvearrowright Y$ and $A$ by $B$, we define $C(H)_{*}$ and $C(H)_{*}^{(2)}$ in an analogous fashion. A similar discussion as before applies; so $C(H)_{*}$ is a finite projective resolution of $L^{\infty}(B)$ over the ring $\chi_{B} L^{\infty}(Y, \mathbb{Z}) * H \chi_{B}$, and

It remains to prove that

$$
\rho^{(2)}\left(C(H)^{(2)}\right)=\frac{\rho^{(2)}(H)}{\mu_{Y}(B)} .
$$

$$
\rho^{(2)}\left(C(G)^{(2)}\right)=\rho^{(2)}\left(C(H)^{(2)}\right) .
$$


Via the ring isomorphism $f_{*}$ we obtain $\chi_{B} L^{\infty}(Y, \mathbb{Z}) * H \chi_{B}$-module structures on $C(G)_{*}$ and indicate this by writing $f_{*}\left(C(G)_{*}\right)$. So $f_{*}\left(C(G)_{*}\right)$ is a finite projective resolution of $L^{\infty}(B)$ over the ring $\chi_{B} L^{\infty}(Y, \mathbb{Z}) * H \chi_{B}$. We have

$$
\rho^{(2)}\left(L^{2}\left(\left.H \curvearrowright Y\right|_{B}\right) \otimes_{\chi_{B} L^{\infty}(Y, \mathbb{Z}) * \chi_{B}} f_{*}\left(C(G)_{*}\right)\right)=\rho^{(2)}\left(C(G)_{*}^{(2)}\right) .
$$

By the fundamental lemma of homological algebra there exists a $\chi_{B} L^{\infty}(Y, \mathbb{Z}) * H \chi_{B}$-linear chain homotopy equivalence

$$
h_{*}: f_{*}\left(C(G)_{*}\right) \rightarrow C(H)_{*} .
$$

Let cone $_{*}\left(h_{*}\right)$ denote the mapping cone of $h_{*}$ [20, Definition 1.33 on p. 35]; each cone ${ }_{i}\left(h_{*}\right)$ is a finitely generated based projective $\chi_{B} L^{\infty}(Y, \mathbb{Z}) * H \chi_{B}$-module. The chain complex $L^{2}\left(\left.H \curvearrowright Y\right|_{B}\right) \otimes_{\chi_{B} L^{\infty}(Y, \mathbb{Z}) * H \chi_{B}}$ cone $_{*}\left(h_{*}\right)$ coincides with the cone of the Hilbert- $\mathcal{N}(H \curvearrowright$ $\left.\left.Y\right|_{B}\right)$-chain map

$$
h_{*}^{(2)}: L^{2}\left(\left.H \curvearrowright Y\right|_{B}\right) \otimes_{\chi_{B} L^{\infty}(Y, \mathbb{Z}) * H \chi_{B}} f_{*}\left(C(G)_{*}\right) \rightarrow C(H)_{*}^{(2)}
$$

induced by $h_{*}$. Proposition 4.1 implies

$$
\begin{aligned}
\rho^{(2)}\left(\operatorname{cone}_{*}\left(h_{*}^{(2)}\right)\right) & =\rho^{(2)}\left(C(H)_{*}^{(2)}\right)-\rho^{(2)}\left(L^{2}\left(\left.H \curvearrowright Y\right|_{B}\right) \otimes_{\chi_{B} L^{\infty}(Y, \mathbb{Z}) * H \chi_{B}} f_{*}\left(C(G)_{*}\right)\right) \\
& =\rho^{(2)}\left(C(H)_{*}^{(2)}\right)-\rho^{(2)}\left(C(G)_{*}^{(2)}\right) .
\end{aligned}
$$

It remains that show that $\rho^{(2)}\left(\operatorname{cone}_{*}\left(h_{*}\right)\right)=0$ : Since $\operatorname{cone}_{*}\left(h_{*}\right)$ is acyclic, there exists a chain contraction $\delta_{*}$. Let us denote the differentials of the $\chi_{B} L^{\infty}(Y, \mathbb{Z}) * H \chi_{B}$-chain complex cone ${ }_{*}\left(h_{*}\right)$ by $c_{*}$ and the induced differentials of the Hilbert $\mathcal{N}(G)$-chain complex $\operatorname{cone}_{*}\left(h_{*}^{(2)}\right)$ by $c_{*}^{(2)}$. Lemma 4.2 tells us that

$$
\rho^{(2)}\left(\operatorname{cone}_{*}\left(h_{*}^{(2)}\right)\right)=\ln \operatorname{det}_{\mathcal{N}\left(\left.H \curvearrowright Y\right|_{B}\right)}\left(\left(c_{*}^{(2)}+\delta_{*}^{(2)}\right)_{\text {odd }}\right)
$$

with $\delta_{*}^{(2)}$ being the chain contraction induced by $\delta_{*}$. Notice that

$$
\left(c_{*}+\delta_{*}\right)_{\text {odd }}: \oplus_{n \in \mathbb{Z}} \operatorname{cone}_{2 n+1}\left(h_{*}\right) \rightarrow \oplus_{n \in \mathbb{Z}} \operatorname{cone}_{2 n}\left(h_{*}\right)
$$

is an isomorphism because $\left(c_{*}+\delta_{*}\right)_{\text {odd }} \circ\left(c_{*}+\delta_{*}\right)_{\text {even }}$ is given by a a lower triangle matrix with 1 on the diagonal (compare [20, Lemma 3.40 on p. 145]). By Lemma 4.5, for every homomorphism of finitely generated based projective $\chi_{B} L^{\infty}(Y, \mathbb{Z}) * H \chi_{B}$-modules, the determinant of the induced morphisms of Hilbert $\mathcal{N}\left(\left.H \curvearrowright Y\right|_{B}\right)$-modules is $\geq 1$. Since the determinant is multiplicative for isomorphisms [20, Theorem 3.14 on p. 128], we conclude that any isomorphism of Hilbert $\mathcal{N}\left(\left.H \curvearrowright Y\right|_{B}\right)$-modules which comes from a $\chi_{B} L^{\infty}(Y, \mathbb{Z}) * H \chi_{B^{-}}$ isomorphism has determinant 1. In particular, we obtain $\operatorname{det}_{\mathcal{N}\left(\left.H \curvearrowright Y\right|_{B}\right)}\left(\left(c_{*}^{(2)}+\delta_{*}^{(2)}\right)_{\text {odd }}\right)=1$ and $\rho^{(2)}\left(\operatorname{cone}_{*}\left(h_{*}^{(2)}\right)\right)=0$.

\section{A cautionary example}

In Gaboriau's proof [13] of the orbit equivalence invariance of $L^{2}$-Betti numbers one encounters the following situation: One obtains a homotopy equivalence between Hilbert $\mathcal{N}(\mathcal{R})$-complexes $C_{*}$ and $D_{*}$ which is not bounded - unless the given orbit equivalence is uniform. To obtain an estimate (and by symmetry an equality) between the $L^{2}$-Betti numbers of these complexes, one constructs increasing sequences of subcomplexes $C_{*}^{(k)} \subset C_{*}$ and $D_{*}^{(k)} \subset D_{*}$ such that for every $n \geq 0$ the closures of $\bigcup_{k} C_{n}^{(k)}$ and $\bigcup_{k} D_{n}^{(k)}$ are $C_{n}$ and $D_{n}$, respectively, and bounded homotopy retracts $C_{*}^{(k)} \rightarrow D_{*}^{(k)}$ for every $k \in \mathbb{N}$. The needed 
continuity property for the $L^{2}$-Betti numbers boils down to the following (easy) continuity property of the von Neumann trace:

Let $\mathcal{A}$ be a finite von Neumann algebra and $M$ a finitely generated Hilbert $\mathcal{A}$-module. Let $f: M \rightarrow M$ be a positive $\mathcal{A}$-morphism and $p_{k}: M \rightarrow M$ a sequence of $\mathcal{A}$-equivariant projections that weakly converge to the identity. Then

$$
\operatorname{tr}_{\mathcal{A}}(f)=\lim _{k \rightarrow \infty} \operatorname{tr}_{\mathcal{A}}\left(f \circ p_{k}\right) .
$$

To be able to drop the uniformity assumption in Theorem 1.10 one would want, among other things, a similar continuity property of the Fuglede-Kadison determinant. The following theorem, whose proof we omit, states such - but with an important, restrictive assumption on the kernels:

Theorem 5.1. Let $f: U \rightarrow V$ be a morphism of finitely generated Hilbert $\mathcal{A}$-modules. Let $p_{k}: U \rightarrow U$ for $k \in \mathbb{N}$ be a sequence of projections with $\operatorname{ker}(f) \subseteq \operatorname{im}\left(p_{k}\right) \subseteq \operatorname{im}\left(p_{k+1}\right)$ that weakly converges to the identity. Then

$$
\operatorname{det}_{\mathcal{A}}(f)=\lim _{k \rightarrow \infty} \operatorname{det}_{\mathcal{A}}\left(f \circ p_{k}\right) .
$$

Next we will show that the condition $\operatorname{ker}(f) \subset \operatorname{im}\left(p_{k}\right)$ is necessary. In our example, $\mathcal{A}$ is $L^{\infty}([0,1])$, and $f$ is the projection onto the second factor $\operatorname{pr}_{2}: L^{2}([0,1]) \oplus L^{2}([0,1]) \rightarrow$ $L^{2}([0,1])$. We drop the subscript $L^{\infty}([0,1])$ in the notation of the trace and the determinant. Obviously, $\operatorname{det}\left(\mathrm{pr}_{2}\right)=1$. Let $\epsilon_{k}$ for $k=1,2, \ldots$ be any sequence of positive real numbers. Consider the morphism of finitely generated Hilbert $L^{\infty}([0,1])$-modules

$$
\begin{gathered}
u_{k}: L^{2}\left(\left[1-2^{1-k}, 1-2^{-k}\right]\right) \rightarrow L^{2}([0,1]) \oplus L^{2}([0,1]) \\
\phi \mapsto\left(\frac{\epsilon_{k}}{\sqrt{1+\epsilon_{k}^{2}}} \cdot \bar{\phi}, \frac{1}{\sqrt{1+\epsilon_{k}^{2}}} \cdot \bar{\phi}\right),
\end{gathered}
$$

where $\bar{\phi} \in L^{2}([0,1])$ is obtained from $\phi$ by extending by zero on the complement of [1$\left.2^{1-k}, 1-2^{-k}\right]$. Consider the morphism of finitely generated Hilbert $L^{\infty}([0,1])$-modules

$$
\begin{gathered}
v_{k}: L^{2}\left(\left[0,1-2^{1-k}\right]\right) \oplus L^{2}\left(\left[0,1-2^{1-k}\right]\right) \rightarrow L^{2}([0,1]) \oplus L^{2}([0,1]) \\
\left(\phi_{1}, \phi_{2}\right) \mapsto\left(\overline{\phi_{1}}, \overline{\phi_{2}}\right) .
\end{gathered}
$$

The morphisms $u_{k}$ and $v_{k}$ are isometric $L^{\infty}([0,1])$-embeddings, and $\operatorname{im}\left(u_{k}\right)$ and $\operatorname{im}\left(v_{k}\right)$ are othogonal to one another. Then

$$
A_{k}=\operatorname{im}\left(u_{k}\right) \oplus \operatorname{im}\left(v_{k}\right) .
$$

is an increasing sequence of closed subspaces. Since

$$
\operatorname{dim}\left(A_{k}\right)=2 \cdot\left(1-2^{1-k}\right)+2^{1-k}-2^{-k} \stackrel{k \rightarrow \infty}{\longrightarrow} 2,
$$

the sequence of projections defined by

$$
p_{k}: L^{2}([0,1]) \oplus L^{2}([0,1]) \rightarrow L^{2}([0,1]) \oplus L^{2}([0,1]), \operatorname{im}\left(p_{k}\right)=A_{k}
$$


weakly converges to the identity. Furthermore, we have

$$
\begin{aligned}
\operatorname{det}\left(\operatorname{pr}_{2} \circ p_{k}\right)=\operatorname{det}\left(\left.\operatorname{pr}_{2}\right|_{A_{k}}\right)= & \operatorname{det}\left(\operatorname{pr}_{2} \circ\left(u_{k} \oplus v_{k}\right)\right) \\
= & \operatorname{det}\left(\left(\operatorname{pr}_{2} \circ u_{k}\right) \oplus\left(\operatorname{pr}_{2} \circ v_{k}\right)\right) \\
= & \operatorname{det}\left(\operatorname{pr}_{2} \circ u_{k}\right) \cdot \operatorname{det}\left(\operatorname{pr}_{2} \circ v_{k}\right) \\
= & \operatorname{det}\left(\frac{1}{\sqrt{1+\epsilon_{k}^{2}}} \cdot \operatorname{id}_{L^{2}\left(\left[1-2^{1-k}, 1-2^{-k}\right]\right)}\right) \\
& \quad \cdot \operatorname{det}\left(\left.\operatorname{pr}_{2}\right|_{\left.L^{2}\left(\left[0,1-2^{1-k}\right]\right) \oplus L^{2}\left(\left[0,1-2^{1-k}\right]\right)\right)}\right) \\
= & \left(\frac{1}{\sqrt{1+\epsilon_{k}^{2}}}\right)^{\operatorname{dim}\left(L^{2}\left(\left[1-2^{1-k}, 1-2^{-k}\right]\right)\right)} \cdot 1 \\
= & \left(\frac{1}{\sqrt{1+\epsilon_{k}^{2}}}\right)^{2^{1-k}-2^{-k}} \\
= & \left(1+\epsilon_{k}^{2}\right)^{-2^{-k-1}} .
\end{aligned}
$$

If we choose $\epsilon_{k}=\sqrt{k^{2^{k+1}}-1}$, then we obtain that

$$
\begin{aligned}
\operatorname{det}\left(\operatorname{pr}_{2} \circ p_{k}\right) & =\frac{1}{k} \text { for } k=1,2 \ldots ; \\
\operatorname{det}\left(\mathrm{pr}_{2}\right) & =1 .
\end{aligned}
$$

\section{REFERENCES}

[1] D. Burghelea, L. Friedlander, T. Kappeler, and P. McDonald, Analytic and Reidemeister torsion for representations in finite type Hilbert modules, Geom. Funct. Anal. 6 (1996), no. 5, 751-859.

[2] A. Connes, Classification of injective factors. Cases $I I_{1}, I I_{\infty}, I I I_{\lambda}, \lambda \neq 1$, Ann. of Math. (2) 104 (1976), no. 1, 73-115.

[3] Jacques Dixmier, von Neumann algebras, North-Holland Mathematical Library, vol. 27, North-Holland Publishing Co., Amsterdam, 1981.

[4] Józef Dodziuk, Peter Linnell, Varghese Mathai, Thomas Schick, and Stuart Yates, Approximating $L^{2}$ invariants and the Atiyah conjecture, Comm. Pure Appl. Math. 56 (2003), no. 7, 839-873. Dedicated to the memory of Jürgen K. Moser.

[5] H. A. Dye, On groups of measure preserving transformation. I, Amer. J. Math. 81 (1959), 119-159.

[6] - On groups of measure preserving transformations. II, Amer. J. Math. 85 (1963), 551-576.

[7] Gábor Elek and Endre Szabó, Hyperlinearity, essentially free actions and $L^{2}$-invariants. The sofic property, Math. Ann. 332 (2005), no. 2, 421-441.

[8] Jacob Feldman and Calvin C. Moore, Ergodic equivalence relations, cohomology, and von Neumann algebras. I, Trans. Amer. Math. Soc. 234 (1977), no. 2, 289-324.

[9] Ergodic equivalence relations, cohomology, and von Neumann algebras. II, Trans. Amer. Math. Soc. 234 (1977), no. 2, 325-359.

[10] Alex Furman, Gromov's measure equivalence and rigidity of higher rank lattices, Ann. of Math. (2) 150 (1999), no. 3, 1059-1081.

[11] - Orbit equivalence rigidity, Ann. of Math. (2) 150 (1999), no. 3, 1083-1108.

[12] - Outer automorphism groups of some ergodic equivalence relations, Comment. Math. Helv. 80 (2005), no. 1, 157-196.

[13] Damien Gaboriau, Invariants $l^{2}$ de relations d'équivalence et de groupes, Publ. Math. Inst. Hautes Études Sci. 95 (2002), 93-150 (French).

[14] _ Examples of groups that are measure equivalent to the free group, Ergodic Theory Dynam. Systems 25 (2005), no. 6, 1809-1827.

[15] Mikhail Gromov, Asymptotic invariants of infinite groups, Geometric group theory, Vol. 2 (Sussex, 1991), London Math. Soc. Lecture Note Ser., vol. 182, Cambridge Univ. Press, Cambridge, 1993, pp. 1295. 
[16] Vaughan Jones and V. S. Sunder, Introduction to subfactors, London Mathematical Society Lecture Note Series, vol. 234, Cambridge University Press, 1997.

[17] T. Y. Lam, Lectures on modules and rings, Graduate Texts in Mathematics, vol. 189, Springer-Verlag, New York, 1999

[18] W. Lück, Approximating $L^{2}$-invariants by their finite-dimensional analogues, Geom. Funct. Anal. 4 (1994), no. 4, 455-481.

[19] Wolfgang Lück, Hilbert modules and modules over finite von Neumann algebras and applications to $L^{2}$-invariants, Math. Ann. 309 (1997), no. 2, 247-285.

[20] — $L^{2}$-invariants: theory and applications to geometry and $K$-theory, Ergebnisse der Mathematik und ihrer Grenzgebiete. 3. Folge. A Series of Modern Surveys in Mathematics [Results in Mathematics and Related Areas. 3rd Series. A Series of Modern Surveys in Mathematics], vol. 44, Springer-Verlag, Berlin, 2002.

[21] Sorin Popa, Deformation and rigidity for group actions and von Neumann algebras, International Congress of Mathematicians. Vol. I, Eur. Math. Soc., Zürich, 2007, pp. 445-477.

[22] Roman Sauer, $L^{2}$-Invariants of groups and discrete measured groupoids, Dissertation, WWU Münster, http://nbn-resolving.de/urn:nbn:de:hbz:6-85659549583.

[23] — $L^{2}$-Betti numbers of discrete measured groupoids, Internat. J. Algebra Comput. 15 (2005), no. $5-6,1169-1188$.

[24] Homological invariants and quasi-isometry, Geom. Funct. Anal. 16 (2006), no. 2, 476-515.

[25] Thomas Schick, $L^{2}$-determinant class and approximation of $L^{2}$-Betti numbers, Trans. Amer. Math. Soc. 353 (2001), no. 8, 3247-3265 (electronic).

[26] Yehuda Shalom, Harmonic analysis, cohomology, and the large-scale geometry of amenable groups, Acta Math. 192 (2004), no. 2, 119-185.

[27] M. Takesaki, Theory of operator algebras. III, Encyclopaedia of Mathematical Sciences, vol. 127, Springer-Verlag, Berlin, 2003. Operator Algebras and Non-commutative Geometry, 8.

[28] Christian Wegner, $L^{2}$-invariants of finite aspherical $C W$-complexes. to appear in manuscripta mathematica.

Mathematisches Institut, Universität Münster, Einsteinstrasse 62, Münster, D-48149, GerMANY

E-mail address: lueck@uni-muenster.de

E-mail address: sauerr@uni-muenster

E-mail address: c.wegner@uni-muenster.de

URL: http://wwwmath.uni-muenster.de/u/lueck/

URL: http://wwwmath.uni-muenster.de/u/sauerr/

$U R L:$ http://wwwmath.uni-muenster.de/reine/inst/lueck/homepages/christian_wegner/wegner.html 